\title{
Autoinhibitory regulation of S100A8/S100A9 alarmin activity locally restricts sterile inflammation
}

\author{
Thomas Vogl, ${ }^{1,2}$ Athanasios Stratis, ${ }^{3}$ Viktor Wixler, ${ }^{4}$ Tom Völler, ${ }^{1}$ Sumita Thurainayagam, ${ }^{1}$ Selina K. Jorch, ${ }^{1}$ Stefanie Zenker, ${ }^{1}$ \\ Alena Dreiling, ${ }^{2}$ Deblina Chakraborty, ${ }^{1}$ Mareike Fröhling, ${ }^{3}$ Peter Paruzel, ${ }^{3}$ Corinna Wehmeyer, ${ }^{3}$ Sven Hermann, ${ }^{2,5}$ \\ Olympia Papantonopoulou, ${ }^{6}$ Christiane Geyer, ${ }^{2,7}$ Karin Loser, ${ }^{2,8,9}$ Michael Schäfers, ${ }^{2,5,9}$ Stephan Ludwig, ${ }^{4,9}$ Monika Stoll,, ${ }^{10}$ \\ Tomas Leanderson, ${ }^{11}$ Joachim L. Schultze, ${ }^{6}$ Simone König, ${ }^{2}$ Thomas Pap, ${ }^{3}$ and Johannes Roth ${ }^{1,2,9}$ \\ IInstitute of Immunology, and ${ }^{2}$ Interdisciplinary Center for Clinical Research, University of Münster, Münster, Germany. ${ }^{3}$ Institute of Musculoskeletal Medicine and ${ }^{4}$ Institute of Molecular Virology, \\ Zentrum für Molekularbiologie der Entzündung (ZMBE), University Hospital Münster, Münster, Germany. ${ }^{5}$ European Institute for Molecular Imaging, University of Münster, Münster, Germany. \\ ${ }^{6}$ Cenomics and Immunoregulation, Life \& Medical Sciences-Institute (LIMES-Institute), University of Bonn, Bonn, Germany. ${ }^{7}$ Department of Clinical Radiology, ${ }^{8}$ Department of Dermatology, \\ ${ }^{9}$ Cells-in-Motion Cluster of Excellence, and ${ }^{10}$ Institute of Human Cenetics, Genetic Epidemiology, University of Muenster, Muenster, Germany. ${ }^{11}$ Department of Experimental Medical Science, \\ Immunology Group, Lund University, Lund, Sweden.
}

Autoimmune diseases, such as psoriasis and arthritis, show a patchy distribution of inflammation despite systemic dysregulation of adaptive immunity. Thus, additional tissue-derived signals, such as danger-associated molecular patterns (DAMPs), are indispensable for manifestation of local inflammation. S100A8/S100A9 complexes are the most abundant DAMPs in many autoimmune diseases. However, regulatory mechanisms locally restricting DAMP activities are barely understood. We now unravel for the first time, to our knowledge, a mechanism of autoinhibition in mice and humans restricting S100-DAMP activity to local sites of inflammation. Combining protease degradation, pull-down assays, mass spectrometry, and targeted mutations, we identified specific peptide sequences within the second calcium-binding EFhands triggering TLR4/MD2-dependent inflammation. These binding sites are free when S100A8/S100A9 heterodimers are released at sites of inflammation. Subsequently, S100A8/S100A9 activities are locally restricted by calcium-induced (S100A8/ $\mathrm{S100A9})_{2}$ tetramer formation hiding the TLR4/MD2-binding site within the tetramer interphase, thus preventing undesirable systemic effects. Loss of this autoinhibitory mechanism in vivo results in TNF- $\alpha$-driven fatal inflammation, as shown by lack of tetramer formation in crossing S100A9-/- mice with 2 independent TNF- $\alpha$-transgene mouse strains. Since S100A8/S100A9 is the most abundant DAMP in many inflammatory diseases, specifically blocking the TLR4-binding site of active S100 dimers may represent a promising approach for local suppression of inflammatory diseases, avoiding systemic side effects.

\section{Introduction}

The term "sterile inflammation" describes inflammatory processes not triggered by microbial agents $(1,2)$, but rather induced by endogenous molecules released during tissue damage or cell stress (3). For these endogenous triggers, the terms alarmins or danger-associated molecular patterns (DAMPs) have been introduced (4). Many alarmins are primarily involved in intracellular homeostasis, but also act as extracellular danger signals triggering inflammation when released by damaged or activated cells $(3,5$, 6). HMGB1, heat shock proteins, and certain S100 proteins belong to this family.

Many chronic inflammatory diseases are driven by systemic autoimmune mechanisms. However, the pattern of sterile inflammation in these diseases frequently shows a local and patchy distribution, e. g., circumscribed inflammatory plaques of psoriasis beside nonaffected skin that can be explained neither by systemic

Authorship note: TV and AS contributed equally to this work. Conflict of interest: The authors have declared that no conflict of interest exists. Submitted: July 29, 2016; Accepted: February 8, 2018

Reference information: / Clin Invest. 2018;128(5):1852-1866.

https://doi.org/10.1172/JCl89867. dysregulation of the adaptive immune system nor by distribution of autoantigens. It is therefore evident that additional tissuederived signals such as alarmins are indispensable for local differences in the clinical manifestation of inflammation. However, molecular mechanisms responsible for local manifestation of inflammatory activity are barely understood.

S100A8 (also known as myeloid-related protein, MRP8) and S100A9 (MRP14) constitute up to $40 \%$ of cytosolic proteins in neutrophils and $5 \%$ in monocytes (7). They are released by activated phagocytes and promote inflammation as endogenous ligands of the TLR4 complex, but molecular models for these interactions are currently missing (8-12). S100A9-deficient mice revealed a pivotal role of these proteins for many inflammatory processes $(13,14)$. S100A8 and S100A9 are the most abundant alarmins during infections, arthritis, allergies, autoimmune diseases, and inflammatory bowel, pulmonary, or cardiovascular diseases (6, 15). S100A8/S100A9 usually exist as heterodimeric complexes (calprotectin) that form heterotetramers in the presence of calcium (16). There are many contradictory reports about the functional activities of S100A8 and S100A9. However, most published work neglects the possibility of functional variability of different S100 complexes. Describing the structural model for S100-TLR4 
interactions, we identified, for what we believe is the first time, an autoinhibitory feedback mechanism for S100 alarmin functions locally restricting their inflammatory properties. S100A8/S100A9 released by activated phagocytes is only temporarily active in the local extracellular microenvironment due to a mechanism of autoinhibition that is induced by calcium-dependent tetramerization of S100A8/S100A9. Oligomer formation of 2 heterodimers blocks the ability of the heterodimer to bind to TLR4/MD2, thus restricting the activity of these alarmins at local sites of inflammation. Loss of this control mechanism results in fatal TNF- $\alpha$-driven inflammation in vivo. These properties define a specific TLR4binding site on the active dimeric form of S100A8/S100A9 as a promising target for a very specific and locally restricted antiinflammatory therapy minimizing systemic side effects.

\section{Results}

S100A8/S100A9 tetramerization abolishes inflammatory activity. S100A8/S100A9 heterodimers associate to (S100A8/S100A9) tetramers in the presence of calcium (16). Surprisingly, both isolated human and murine S100A8 and S100A9 homodimers induced a strong TNF- $\alpha$ release in human monocytes, whereas the S100A8/S100A9 heterocomplexes were inactive (Figure 1, A and B, and Supplemental Figure 1A; supplemental material available online with this article; https://doi.org/10.1172/JCI89867DS1).

The aa exchange of N69A or E78A in the calcium-binding EF-hand II of S100A9 prevents tetramer formation (17). In contrast with WT (S100A8/S100A9) ${ }_{2}$ tetramers, mutated S100A8/ S100A9(N69A) and S100A8/S100A9(E78A) heterodimers significantly induced TNF- $\alpha$ release in human monocytes (Figure $1 \mathrm{C}$ and Supplemental Figure 1B). Size-exclusion chromatography (SEC) revealed that, in contrast with WT S100A8/S100A9 complexes, mutated heterodimers and S100A8 and S100A9 homodimers did not form tetramers in the presence of calcium (Figure 1D). Thus, loss of biological activity is strictly linked to calciuminduced tetramer formation, representing a mechanism of autoinhibition of S100 activity (Figure 1E). Finally, global geneexpression analysis confirmed that S100A8 or S100A9 homodimers, but not S100 tetramers, induced a global transcriptional response that was basically identical to that of LPS-mediated TLR4 stimulation (Figure $1 \mathrm{~F}$ and Supplemental Table 1), confirming that activity of S100A8/S100A9 is restricted to the dimeric state. In the following experiments, we used S100A9 homodimers as a model for the in-depth analysis of dimermediated effects in vitro. Using transfected HEK293 cells, we confirmed that S100A9 dimer-induced activation was dependent on a functional TLR4-MD2-CD14 complex (Supplemental Figure 1C). Accordingly, blocking TLR4 with the monoclonal antibody HTA125 or TLR4 antagonist RS-LPS efficiently inhibited S100A9-induced TNF- $\alpha$ expression (Supplemental Figure 1D). Calcium-induced tetramers of S100A8/S100A9 were unable to block LPS or S100A8 homodimer-dependent TLR4/ MD2 activation of human monocytes (Supplemental Figure 1E). Furthermore, S100A9 dimers induced TLR4-MD2 downstream signaling, activating MyD88, IRAK, PKC, ERK1/2, p38 kinases, and $\mathrm{NF}-\kappa \mathrm{B}$ (Supplemental Figure 2, A-E).

S100A 8 dimers amplify TNF- $\alpha$-mediated inflammation. $\mathrm{KO}$ of S100A9, which is generally associated with a loss of S100A8 at the protein level $(13,14)$, protects against overwhelming inflammation in endotoxin-induced shock, at least partly due to the lower expression of TNF- $\alpha$ (8). To answer the question of whether S100 proteins act up- or downstream of TNF- $\alpha$-driven inflammation, we analyzed tristetraprolin-KO mice $\left(\mathrm{TTP}^{-/-}\right)$constitutively expressing high levels of murine TNF- $\alpha$. $\mathrm{TTP}^{-/-}$mice appear normal at birth, but develop erosive arthritis and dermatitis during the first month of life $(18,19)$. Unexpectedly, $\mathrm{TTP}^{-/-} \mathrm{S}_{100 \mathrm{~A}} 9^{-/-}$mice did not improve in their symptoms, but on the contrary, started to develop severe psoriasis-like skin inflammation, characterized by keratinocyte hyperproliferation and widespread scaling within the first days after birth (Figure 2, A and B), as reflected by an increase of an adapted Psoriasis Area and Severity Index (PASI) (20) (Figure 2A). Compared with $\mathrm{TTP}^{-/-}$mice, $\mathrm{TTP}^{-/-} \mathrm{S} 100 \mathrm{A9}^{-/-}$animals showed a markedly thickened epidermis (acanthosis), loss of the granular layer, extension of the cornified envelope as a result of incomplete keratinization (parakeratosis), elongation of rete ridges, and a typical hyperpigmentation of the basal layer (Figure 2, B and C). Ki67 staining revealed a hyperproliferation of keratinocytes in all skin layers of $\mathrm{TTP}^{-/-}$S100A9 $9^{-/-}$mice, which is known as a hallmark of psoriatic lesions. In contrast, the expression of keratin 10 (K10) and the terminal differentiation marker loricrin (Lor) was markedly reduced in $\mathrm{TTP}^{-/-}$S100A9 ${ }^{-/-}$mice versus controls, indicating incomplete keratinocyte differentiation. As typical for psoriasiform dermatitis, $\mathrm{TTP}^{-/-} \mathrm{S} 100 \mathrm{~A} 9^{-/-}$mice showed dermal invasion of macrophages, granulocytes, and $\mathrm{T}$ cells as well as subcorneal accumulation of invading granulocytes, i.e., Munro microabscesses (Figure 2B).

In accordance with this psoriasis-like phenotype, we detected significantly increased levels of IFN- $\gamma$, IL-6, IL-17, IL-22, IL-23, and IL-36 (21-23) in the skin of $\mathrm{TTP}^{-/-}$S100A9 ${ }^{-/-}$mice versus controls (Figure 2, D and E). Although S100A9 ${ }^{-/-}$mice have been shown to constitute a functional double-KO for both S100 proteins under steady-state as well as under acute inflammatory conditions $(13,14)$, we detected, for what we believe is the first time, a strong recovery of S100A8 protein expression under chronic TNF- $\alpha$ expression in the hyperproliferative epidermis of $\mathrm{TTP}^{-/}$ S100A9 ${ }^{-/-}$mice compared with the skin of $\mathrm{TTP}^{-/}$, $\mathrm{S}_{100 A 9}{ }^{-/}$, or WT mice (Figure 2B).

Blocking TNF- $\alpha$ improves psoriasis-like skin disease in TTP S10OA9 $/$ mice. Depletion of TNF- $\alpha$ by treatment of newborn $\mathrm{TTP}^{-/-}$S100A9 ${ }^{-/-}$mice with anti-TNF- $\alpha$ antibodies (certolizumab) led to a significant amelioration of disease activity, as evidenced by decreased PASI scores, keratinocyte hyperproliferation, and reduced scaling of the skin (Figure 3, A and B). In parallel, S100A8 as well as cytokine expression was markedly reduced in the epidermis of anti-TNF- $\alpha$-treated $\mathrm{TTP}^{-/-}$S100A9 ${ }^{-/-}$mice (Figure 3, B-D). In accordance with the TNF- $\alpha$-blocking studies, $\mathrm{TTP}^{-/-}$S100A9-/mice on a TNF- $\alpha$-deficient background showed a complete absence of psoriasis-like skin inflammation. Moreover, the lack of TNF- $\alpha$ reduced S100A8 protein expression in the skin to the level of that in healthy WT mice (Supplemental Figure 3). Finally, isolated keratinocytes from S100A9-/- mice showed a clear upregulation of S100A8 protein expression after TNF- $\alpha$ stimulation by Western blot analysis (Supplemental Figure 4A).

S1OOA 8 protein recovery in ihTNF- $t g$ S100A9 ${ }^{-/-}$mice promotes joint and skin inflammation. To exclude nonspecific effects of 
A
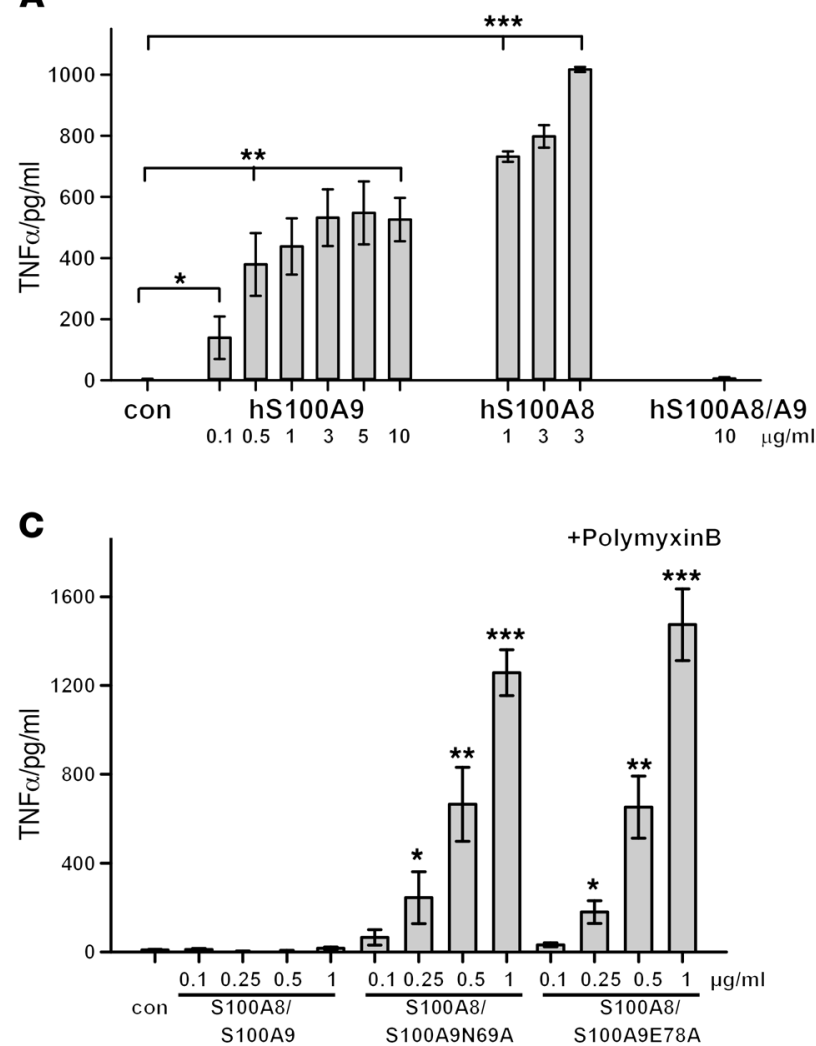

$\mathbf{E}$

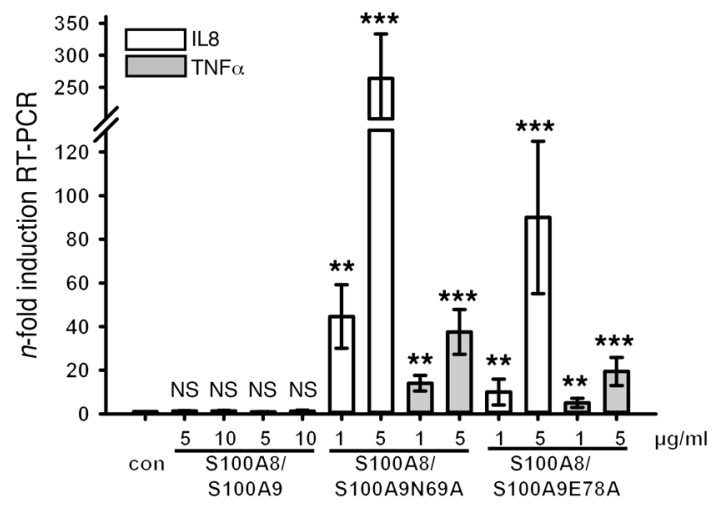

B
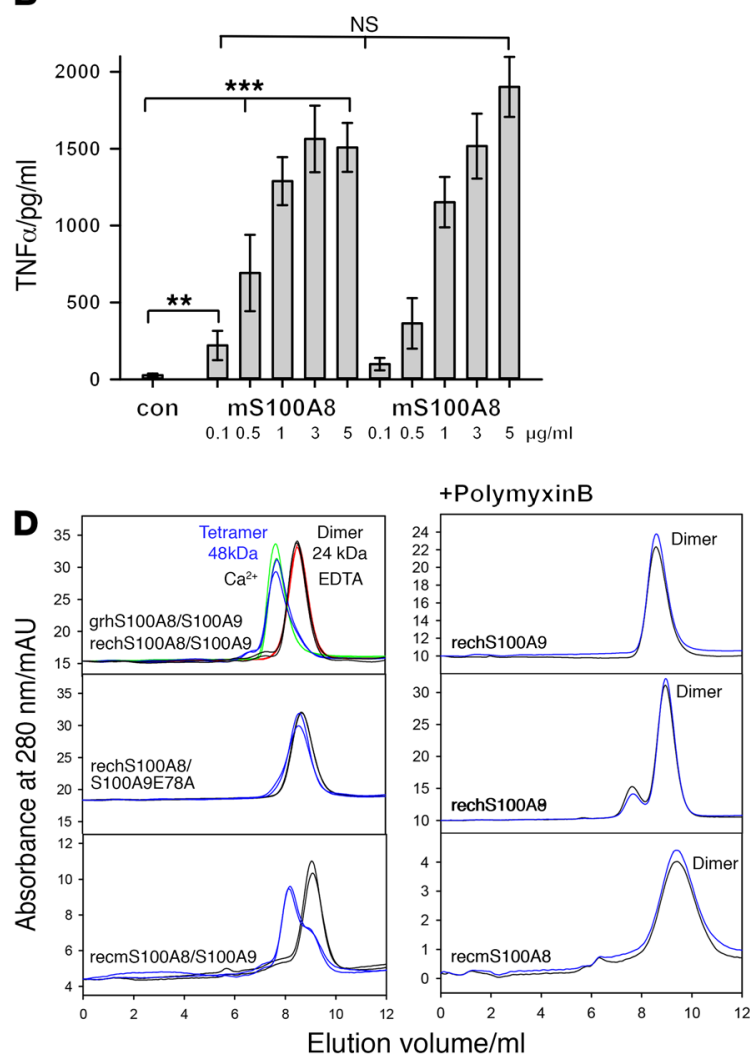

$\mathbf{F}$

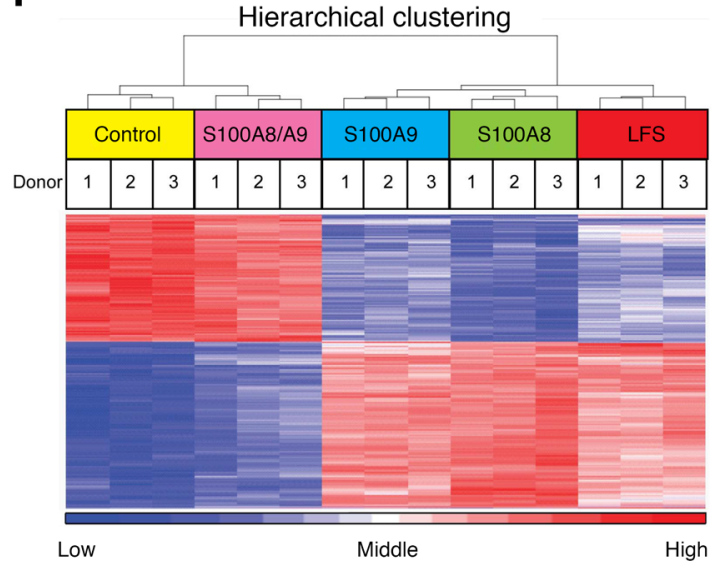

Figure 1. S100A8/S100A9 tetramerization blocks inflammatory activity of the alarmin. (A and B) Effects of hS100A9 and hS100A8 homodimers or hS100A8/S100A9 (A), mS100A8 homodimer (B), or hS100A8/S100A9, hS100A8/S100A9N69A, and hS100A8/S100A9E78A (C) on TNF- $\alpha$ production by monocytes quantified by ELISA after 4 hours of incubation ( $>1 \mathrm{mM}$ calcium in culture medium). (D) Representative SEC runs of human (grhS100A8/ S100A9, granulocytic prepared human S100A8/S100A9 [red and green curves]; rechS100A8/S100A9, recombinant prepared human S100A8/S100A9; rechS100A8/S100A9E78A, recombinant prepared human S100A8/S100A9E78A; rechS100A8, recombinant prepared human S100A8; rechS100A9, recombinant prepared human S100A9) and murine (recmS100A8/S100A9, recombinant prepared murine S100A8/S100A9;recmS100A8, recombinant prepared murine S100A8) S100 proteins in the absence (black and red curves) and presence of $100 \mu \mathrm{M} \mathrm{Ca2}{ }^{+}$ions (blue and green curves) ( 3 independent experiments). (E) Induction of TNF- $\alpha$ and IL-8 mRNA in HEK293 cells expressing TLR4-MD2-CD14 and treated with either 5 or $10 \mu \mathrm{g} / \mathrm{ml}$ of rhS100A8/S100A9 (left), 1 or $5 \mu \mathrm{g} / \mathrm{ml}$ rhS100A8/S100A9N69A (middle), or 1 or $5 \mu \mathrm{g} / \mathrm{ml} \mathrm{hS100A8/S100A9E78A} \mathrm{(right)} \mathrm{for} 4$ hours, as detected by qRT-PCR. Results are shown as relative to baseline expression in unstimulated cells. RPL was used as a housekeeping control gene. (F) Human monocytes were left untreated or stimulated with $5 \mu \mathrm{g} / \mathrm{ml}$ of either S100A8/S100A9, S100A9, or S100A8 or $1 \mathrm{ng} / \mathrm{ml}$ LPS for 4 hours. RNA was isolated and used for transcriptome analyses. The 1,000 most variable genes (FDR corrected, $P<0.05$ ) within the data set were used for HC. Data were $Z$ score-normalized and ranked according to changes in expression upon stimulus. Data represent mean $\pm \mathrm{SD}$ of 5 independent experiments. ${ }^{*} P<0.05 ;{ }^{* *} P<0.01 ;{ }^{* * *} P<0.001,2$ tailed $t$ test. 
A

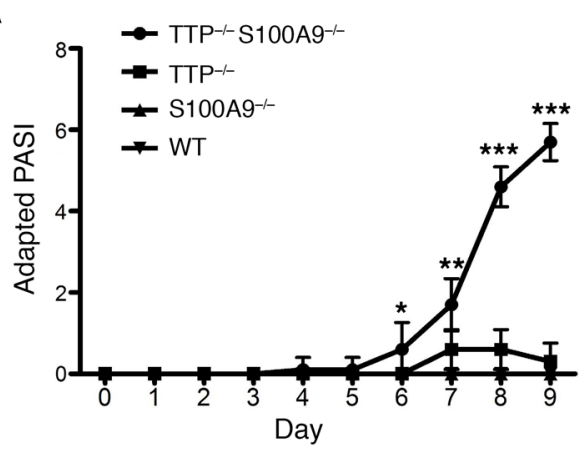

C

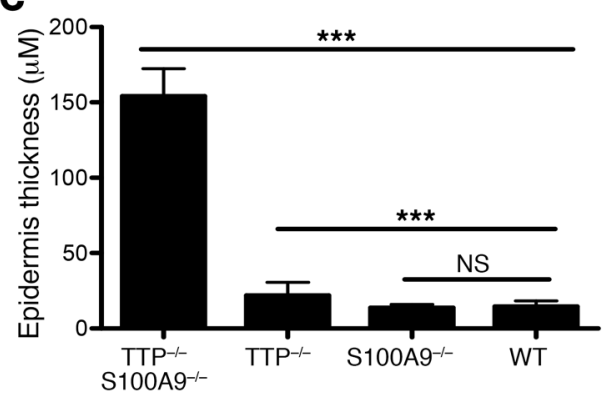

D

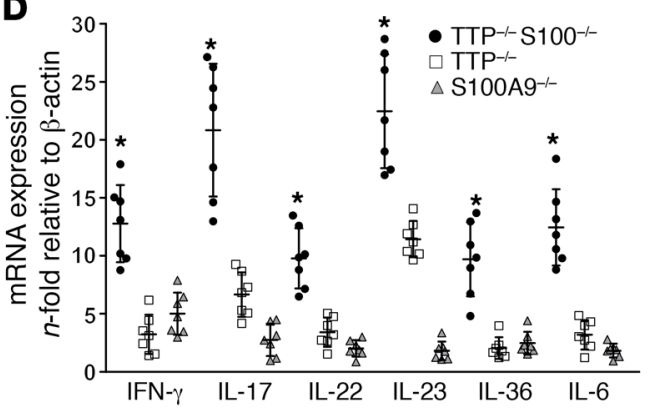

E

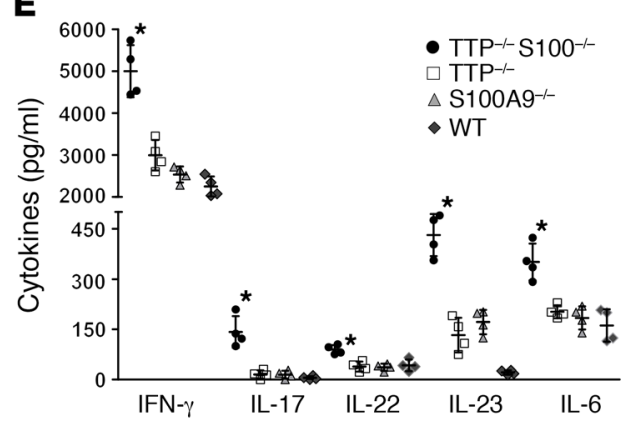

B
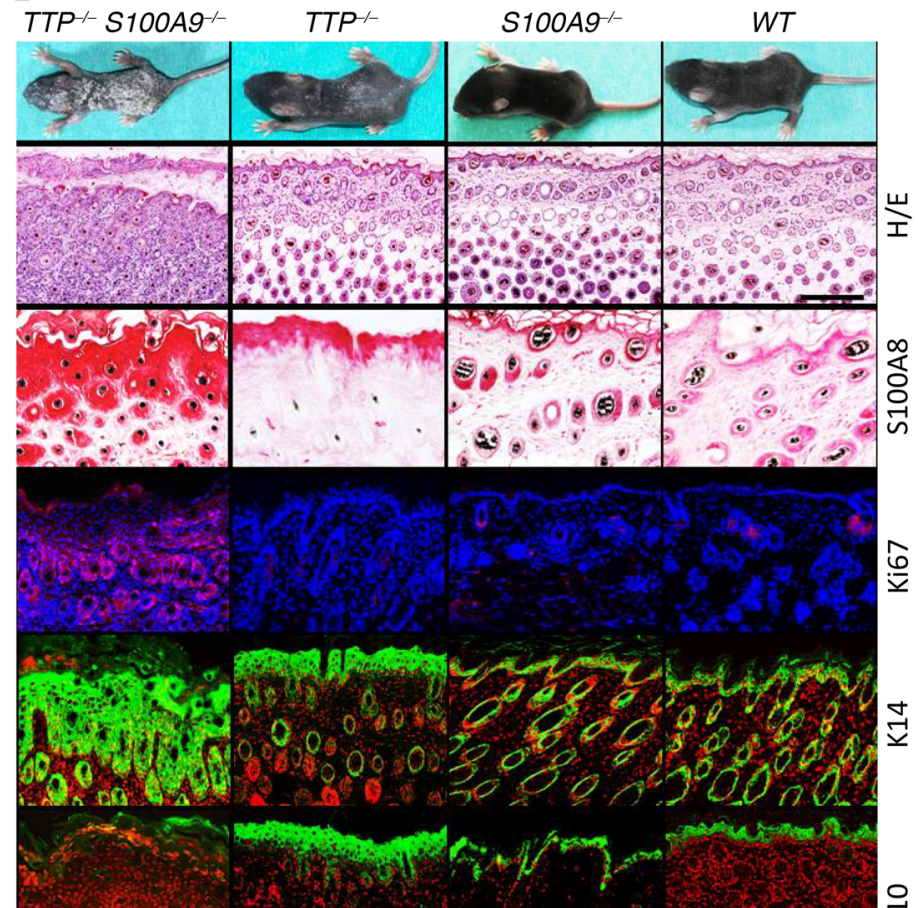

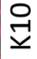

Figure 2. Phenotype of TTP-/- S100A9-/- mice. (A) Disease progression of TTP ${ }^{-/-}$S100A9 ${ }^{-/-}$mice versus controls (10 mice per group) is shown by the adapted PASI scores from P1-P9. ${ }^{*} P<0.05$; ${ }^{* *} P<0.01$; ${ }^{* *} P<0.001$, Mann-Whitney $U$ test. (B) Photographs and skin sections of TTP-/- S100A9 ${ }^{-/-}$, TTP ${ }^{-/-}$, S100A $9^{-/-}$, and WT mice at day 9 after birth. Sections were stained with H\&E or by immunohistochemistry for S100A8 or immunofluorescence for the indicated epidermal differentiation markers or invading T cells (CD3), granulocytes (Gr1), and macrophages (F4/80) (fluorescence, green signal) and Ki67 (fluorescence, red signal). Blue and red counterstaining shows nuclei. Scale bars: $200 \mu \mathrm{m}$ (H\&E); $50 \mu \mathrm{m}$ (immunostaining). (C) Epidermis thickness was determined for at least 3 sections per mouse and for each section at 3 different sites and at least 5 mice for each group. (D and $\mathbf{E}$ ) Expression of IFN- $\gamma$, IL-6, IL-17, IL-22, IL-23, and IL-36 in the skin of TTP ${ }^{-1-}$ S100A9-/- mice at day 9 after birth compared with TTP ${ }^{-/-}$and S100A9-/- mice. The mRNA levels in the skin were measured by qRT-PCR (D) and quantified at the protein level by FACS-based analysis (E) of 3 independent experiments. Data represent mean \pm SEM. ${ }^{*} P<0.05 ;{ }^{* *} P<0.01 ;{ }^{* *} P<0.001,1$-way ANOVA (C-E).

TTP KO $(24,25)$, we crossed S100A9-/- mice with the ihTNF-tg mouse strain, which displays features of psoriatic arthritis 3 to 6 weeks after TNF- $\alpha$ induction (26). Expression of human TNF- $\alpha$ was induced in adult mice by doxycycline (dox) for up to
8 weeks. Disease progression in ihTNF-tg S100A9-/- mice was significantly aggravated compared with that in ihTNF-tg mice, as shown by loss of body weight and grip strength as well as increased paw swelling. None of the control mice developed any 
A
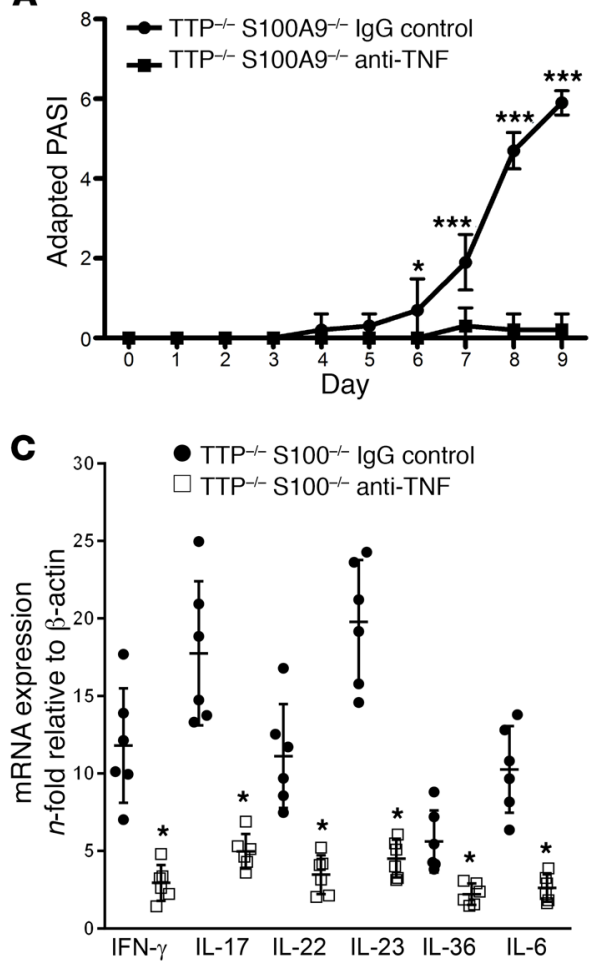

D

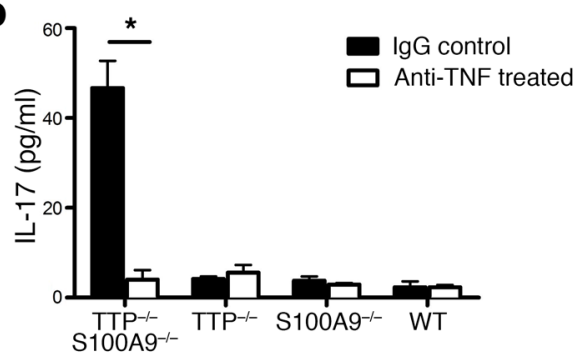

B

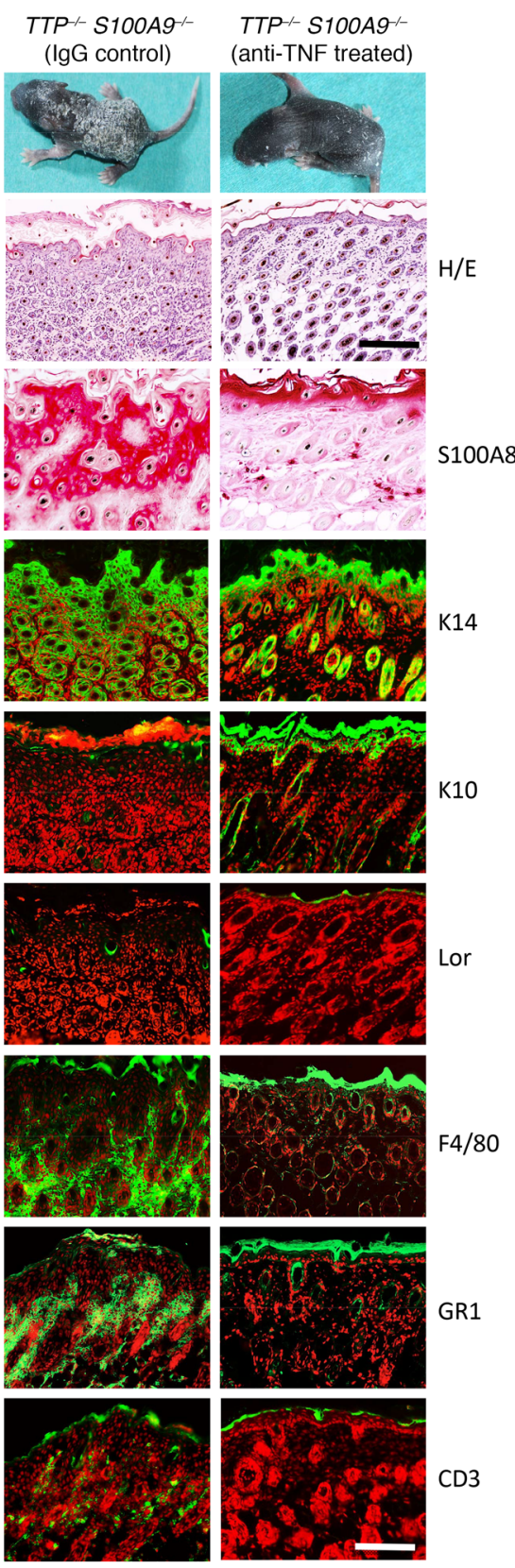

Figure 3. Improvement of psoriasis-like skin disease in $\mathrm{TTP}^{-/-}$S100 A9 ${ }^{-/-}$mice after treatment with anti-TNF- $\alpha$. (A) Adapted PASI score of anti-TNF- $\alpha$-treated mice (40 mg certolizumab/kg mouse on days 4,6 , and 8) shows amelioration of disease progression compared with IgC control antibody-treated mice (10 mice for each group). ${ }^{*} P<0.05$; ${ }^{* *} P<0.001$, MannWhitney $U$ test. (B) Photographs of mice and immunostainings of skin sections from anti-TNF- $\alpha$-treated (right panel) and IgG-treated (left panel) TTP ${ }^{-1-}$ S100 $\mathrm{Ag}^{-/}$ mice at day 9 after birth. Sections were stained with $\mathrm{H} \& \mathrm{E}$ or with antibodies against the indicated epidermal differentiation markers or with antibodies against the immune cell markers F4/80, Gr1, and CD3 (green signal). Red counterstaining shows nuclei. Scale bars: $200 \mu \mathrm{m}$ (H\&E); $50 \mu \mathrm{m}$ (immunostaining). (C) mRNA levels of IFN- $\gamma$, IL-6, IL-17, IL-22, IL-23, and IL-36 in the skin of anti-TNF- $\alpha$ and IgG-treated $\mathrm{TTP}^{-/-}$S100A9 - $^{-/}$mice were measured by qRT-PCR. (D) Expression of IL-17 protein was analyzed in lesional skin by FACSbased protein quantification from antiTNF- $\alpha$ - and IgG-treated TTP- ${ }^{-1-}$ S100A9- ${ }^{-1-}$ mice. Data represent mean \pm SEM from 3 independent experiments. ${ }^{*} P<0.05$; ${ }^{* *} P<0.001$, 1-way ANOVA (C and $\left.\mathbf{D}\right)$. abnormalities after dox application (Figure 4, A-C). Employing optical imaging (OI) (fluorescence reflectance imaging [FRI]) using Cy5.5-labeled anti-S100A8 antibodies (27), we confirmed the recovery of S100A8 expression in vivo after TNF- $\alpha$ induction. Significant local tracer accumulation was observed in doxtreated ihTNF-tg S100A9-- mice in paws and knee joints as well as in the skin (Figure 4D). The recovery of the S100A8 protein in ihTNF-tg S100A9-/- mice after dox stimulation was also confirmed by immunohistochemistry in the skin (Figure 4E), nail matrix (Figure $4 \mathrm{~F}$ ), and bone marrow as well as in bone marrowcontaining metacarpal sections (Figure $4 \mathrm{G}$ and Supplemental Figure 5) and blood cells (Figure 4H). Histological analyses of distal interphalangeal (DIP) joints revealed a stronger hyperplasia of synovial tissue and cartilage destruction in ihTNF-tg S100A9-/- mice compared with that in ihTNF-tg mice (Supple- mental Figure 6). Prolonged stimulation of isolated S100A9-1bone marrow cells (BMCs) with TNF- $\alpha$ induced a recovery of S100A8 homodimers on the protein level, as observed before for S100A9-/- keratinocytes (Supplemental Figure 4B). In addition, S100A8 was released into the supernatant (Supplemental Figure 4B). In the absence of prolonged TNF- $\alpha$ stimulation, S100A8 homodimers were rapidly degraded in the proteasome (Supplemental Figure 4C). Taken together, these data demonstrate a strong induction of the S100A8 protein after prolonged TNF- $\alpha$ expression in the absence of S100A9 in vivo. As shown above, S100A8 cannot form tetramers in the absence of S100A9. Lack of this autoinhibitory mechanism results in expression of dominant active S100A8 dimers, which drive uncontrolled and, in the case of TTP KO, even fatal inflammation. Healthy $\mathrm{S} 100 \mathrm{~A}^{-/ /}$mice are protected from fatal inflammatory reactions 
A

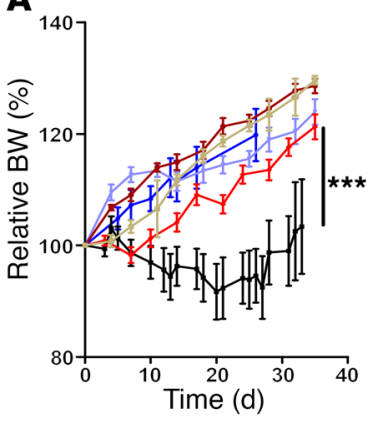

B

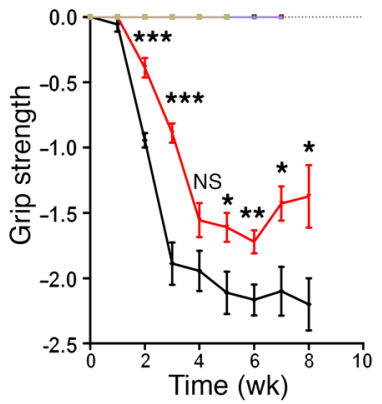

C

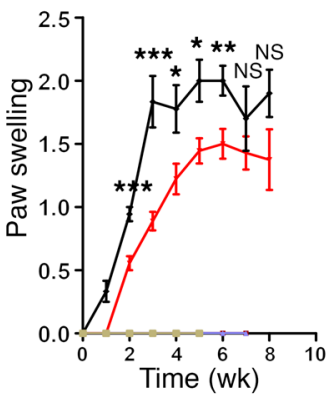

- inTNFtg S100A9--1 w/o dox

- inTNFtg S100A9 $9^{-/-}+$dox

- inTNFtg w/o dox

- inTNFtg + dox

$\rightarrow$ S100A9-1- W/o dox

- S100A9 ${ }^{-1-}+$ dox

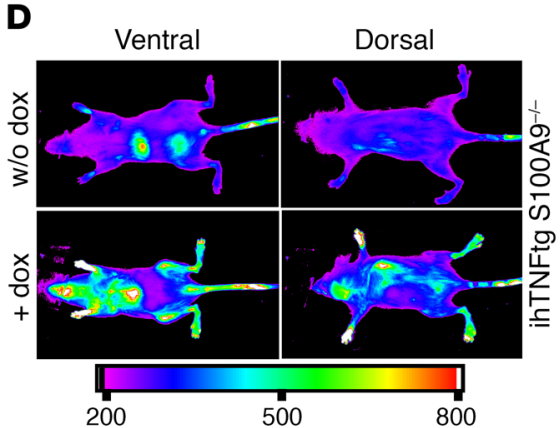

E

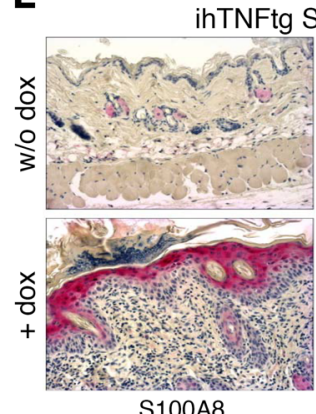
S100A8

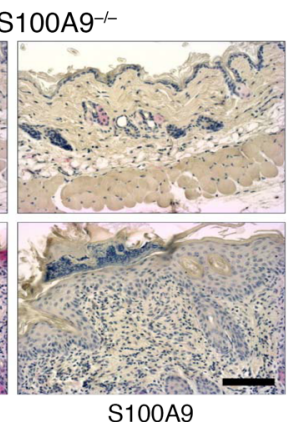

S100A9
$\mathbf{F}$

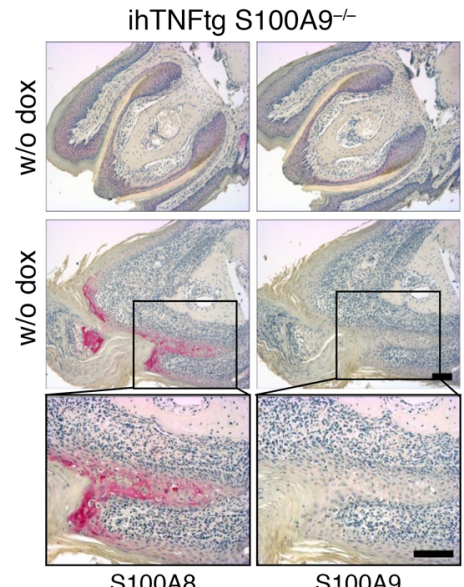

G

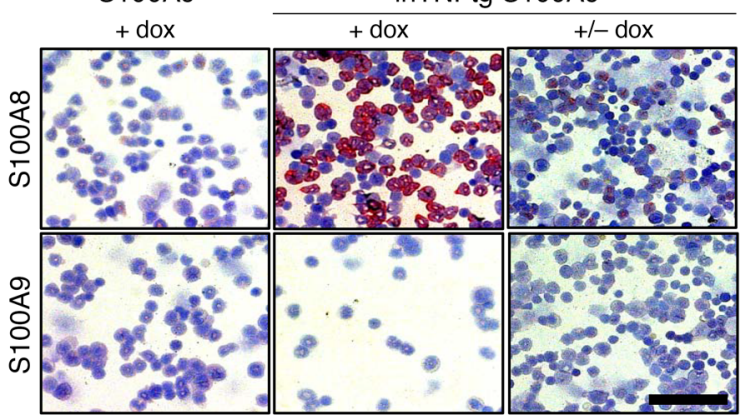

H

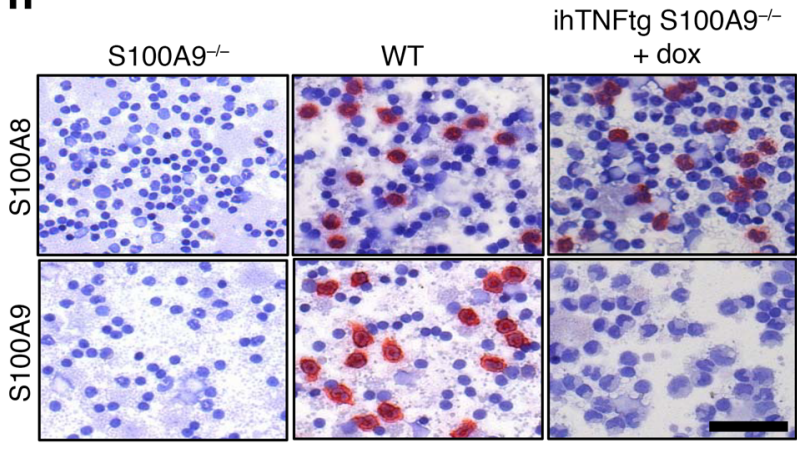

Figure 4. S100A8 recovery aggravates disease progression in ihTNF-tg S100A9-/- mice. (A-C) Changes in body weight (A), grip strength (B), and swelling of forepaws (C) of dox-treated ihTNF-tg S100A9-/- mice compared with ihTNF-tg, S100A ${ }^{-1-}$, or control mice. Data are shown as mean \pm SEM of 3 independent experiments. $n=6$ mice per group in each experiment. ${ }^{*} P<0.05$; ${ }^{*} P<0.01$; ${ }^{* * *} P<0.001$, 1-way ANOVA. (D) Control (upper images) or doxtreated (lower images) ihTNF-tg S100A ${ }^{-1-}$ mice ( $n=5$ for each group) were injected with Cy5.5-labeled anti-S100A8, and whole-body OI was recorded by FRI 24 hours later. Strong fluorescence intensities were detected in all paws and skin, indicating S100A8 protein recovery during chronic TNF- $\alpha$ stimulation. Nonspecific background signals in the liver and bladder indicate tracer degradation. (E and F) S100A8 and S100A9 immunostainings of skin (E) and forepaw toes (F) of inTNF-tg S100A9-/- mice in the presence (6 weeks) or absence of dox confirm S100A8 recovery in the nail matrix, skin keratinocytes, and phagocytes. The lower 2 images in $\mathbf{F}$ are magnifications of boxes in middle images. Scale bars: $100 \mu \mathrm{m}$. (G, $\mathbf{H})$ Cytospin preparations of bone marrow (C) and blood cells (H) of ihTNF-tg S100A9-/- or S100A9-/- mice treated with dox for 6 (G) or 3 weeks (H). Cells were stained for S100A8/S100A9 expression. Scale bars: $50 \mu \mathrm{m}$. In contrast with S100A9 ${ }^{-/-}$mice, ihTNF-tg S100A9-/- animals showed a pronounced recovery of S100A8 protein in BMCs after dox stimulation (middle panel), which was abolished after removal of dox for 7 days (right panel). (H) Cytospin preparations of blood cells from dox-treated

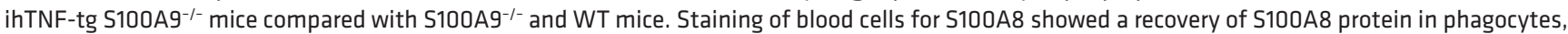
but not lymphocytes, after TNF- $\alpha$ induction.

due to appropriate proteasome degradation over time, while this mechanism is inadequate during chronic TNF- $\alpha$ conditions.

Identification of the S100A9-binding site on TLR4/MD2. Structural analysis revealed several aa hidden in the (S100A8/ S100A9) ${ }_{2}$ tetramer interface (Figure 5A), but freely accessible in the heterodimer (Figure 5B) (28), most of which were associated with the second calcium-binding EF-hands of both S100 subunits. We changed selected aa of S100A9 (E64, D65, K72, Q73, E77, and R85) to alanine as a model of active S100 dimers (29).
These aa were not involved in the coordination of the calcium ion, showed little contact with neighboring aa, and were freely accessible in the dimer (Figure 5C). Four of the six exchanges in S100A9 (E64A, D65A, Q73A, and E77A) already showed a trend in binding reduction of about 20\% compared with WT S100A9 homodimers in a quantitative TLR4/MD2-binding assay (Figure 5D). However, by changing 2 of the selected aa, S100A9 dimers lost their affinity to the TLR4/MD2 complex almost completely (Figure 5E). Changes in the overall structure of the mutated 


\section{Table 1. Putative contact residues of S100A9 homodimer and TLR4/MD2}

\begin{tabular}{|c|c|c|c|}
\hline $\begin{array}{l}\text { Residues in } \\
\text { S100A9 }\end{array}$ & $\begin{array}{l}\text { Residues in } \\
\text { S100A9 }\end{array}$ & $\begin{array}{l}\text { Residues in } \\
\text { TLR4 }\end{array}$ & $\begin{array}{l}\text { Residues in } \\
\text { MD2 }\end{array}$ \\
\hline VAL 24 & & HIS 431 & \\
\hline ASP 30 & & & LYS 89 \\
\hline \multirow[t]{3}{*}{ CLU 39} & & LYS 435 & \\
\hline & LYS 50 & ALA 610, GLN 616 & \\
\hline & LYS 54 & SER 613, ASP 614 & \\
\hline ASP 65 & & & LYS 125 \\
\hline \multirow[t]{2}{*}{ THR 68} & & & TYR 131 \\
\hline & THR 68 & & THR 84 \\
\hline ASP 71 & & & ARG 90 \\
\hline LYS 72 & & & GLY 123 \\
\hline GLN 73 & & & ARG 90 \\
\hline \multirow[t]{2}{*}{ GLU 77} & & & ASN 86 \\
\hline & ARG85 & & THR 84 \\
\hline
\end{tabular}

proteins were excluded by fluorescence emission spectrometry (Supplemental Figure 7A).

After almost complete trypsin digestion of S100A9 (Figure $5 \mathrm{~F}$, insert), half of the inflammatory activity on monocytes was still present, indicating that a peptidic fragment or fragments of S100A9 are sufficient to activate monocytes (Figure 5F). Using this preparation, 1 specific peptide (peptide no. 15 of 17 possible peptides without missed cleavages) was detected by a TLR4/MD2 pull-down assay and identified by mass spectrometry analysis, revealing the sequence of S100A9 from aa 73 to 85 (Figure 5G), which is almost identical to that of the peptide identified by structural analysis and site-directed mutagenesis described above. We next synthesized a peptide comprising the whole EF-hand II of S100A9 (peptides 63-79, MEDLDTNADKQLSFEEF) and confirmed its specific binding to TLR4/MD2, while a mutated control peptide (63-79 5A, MAALDTNADAALSFAEF; bold letters indicate introduced mutations) or a scrambled peptide sequence (DSLEMTEENLADQFKDF) failed to bind to the receptor complex completely (Figure $5 \mathrm{H}$ ). S100A9 peptides with only 2 mutations in the sequence (MEALDTNADKALSFEEF or MADLDTNADKQLSFAEF) showed reductions in binding properties similar to those observed for homologues mutations in the S100A9 fulllength protein (Supplemental Figure 7B).

Model of the S10OA9 homodimer interaction with TLR4/MD2. Using the known coordinates of the solved 3D structures for the human S100A9 dimer (Research Collaboratory for Structural Bioinformatics [RSCB] PDB ID: 1IRJ; https://www.rcsb.org/) and TLR4/MD2 (PDB ID: 3FXI), we simulated the binding of the synthesized peptide corresponding to EF-hand II of the S100A9 subunit with MD2 (Figure 6A). Unbiased studies of the docking of the S100A9 homodimer to TLR4/MD2 (Figure 6, B and C) confirmed this peptide-MD2 interaction for the intact S100A9 homodimer as well and identified additional aa potentially involved in binding to TLR4/MD2 (Table 1). With the exception of amino acid $64(\mathrm{Glu})$, we were able to assign specific interaction residues for all aa mutated in S100A9 (D65, K72, Q73, E77, and
R85) in MD2, including arginine 85 (second S100A9 subunit). Structural modeling of the S100A8/S100A9 heterodimer (PDB ID 1XK4, chains $\mathrm{E}$ and $\mathrm{G}$ ) with TLR4/MD2 resulted in a model close to the homodimeric S100A9/TLR4/MD2 model (Figure 6, $\mathrm{B}$ and $\mathrm{D})$. In a granuloma mouse model, we finally confirmed the presence of active dimers in vivo by crosslinking noncovalently associated S100A8/S100A9 complexes (Supplemental Figure 8, A and B). Finally, tetramer formation of S100A8/S100A9 heterodimers was significantly diminished under acidic conditions typical for inflammatory tissues/fluids (starting at $\mathrm{pH}$ values below 5.8; Supplemental Figure 8, C-E).

\section{Discussion}

The concept of sterile inflammation driven by endogenous danger signals has markedly increased our understanding of numerous inflammatory diseases. S100A8/S100A9 are the most abundant alarmins in many clinically relevant disorders, such as rheumatoid arthritis or other autoimmune diseases, inflammatory lung or bowel diseases, allergies, or cardiovascular complications $(3,6)$. Although the alarmin concept is supported by different gene KO models in mice, clear mechanistic models of their local regulation are lacking. We now demonstrate an autoinhibitory mechanism in which calcium-induced tetramer formation abolishes the proinflammatory activity of S100A8/S100A9 dimers, which has several implications for the field of alarmin-driven inflammation in general and S100 biology in particular. Our data unravel a model of a local self-control mechanism of $\mathrm{S100}$ alarmin-driven inflammation. In the intracellular low-calcium milieu, S100A8 and S100A9 are present as heterodimers that are secreted by activated phagocytes at the site of inflammation (30-32). These dimers interact with and activate TLR4/MD2-expressing cells via a specific binding site mainly located in the second EF-hand, thus promoting inflammatory processes. Due to high extracellular calcium concentrations, heterodimers subsequently associate to $(\mathrm{S100A8/S100A9)})_{2}$ tetramers. Tetramers are unable to bind to TLR4/MD2, thereby restricting the biological activity of $\mathrm{S} 100$ proteins locally in order to avoid undesirable systemic inflammatory side effects. The binding of other TLR4/MD2 agonists, including LPS and S100 homodimers, is unaffected by tetramer formation. Our findings explain many contradictory reports regarding inflammatory $\mathrm{S} 100$ effects, since most studies did not consider the fact that different complex forms may show different inflammatory activities (33). The biological relevance of our hypothesis was confirmed in 2 independent TNF- $\alpha$-driven mouse models of inflammation. In both of these models, S100A9 deficiency promoted uncontrolled inflammation due to S100A8 dimer recovery. The presence of dominant active S100A8 dimers prevented calcium-induced tetramer formation and controlled the inflammatory effects by autoinhibition due to the absence of the S100A9 subunit. Analysis of protein samples directly obtained from phagocytes, serum, or other biological samples indicated that S100A8/S100A9 heterocomplexes are the predominant form of these molecules in vivo (33). This assumption is supported by experimental evidence analyzing S100A8/S100A9 interactions in vivo (e. g., yeast 2-hybrid system) (34) and comparative biophysical analysis of homo- and heterocomplexes (35) as well as structural data $(28,29,36)$, indicating that homodimers are a useful 
A

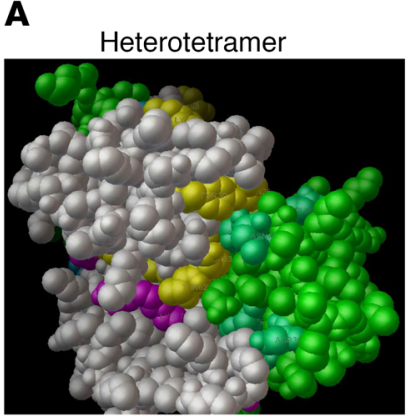

D

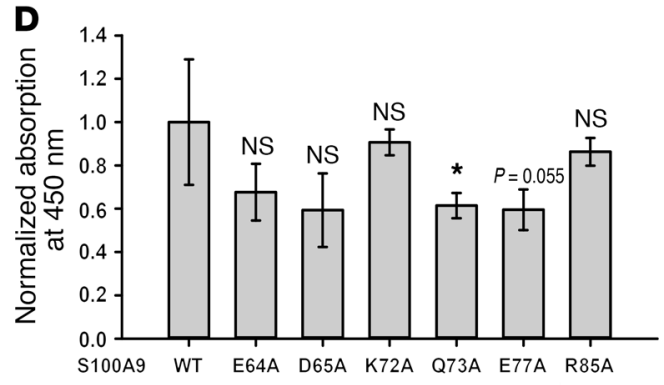

$\mathbf{F}$

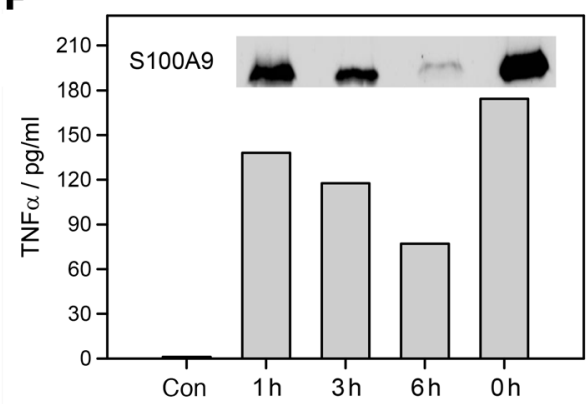

G

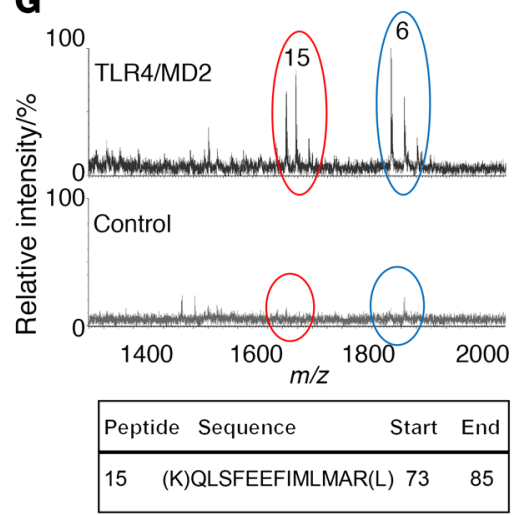

B

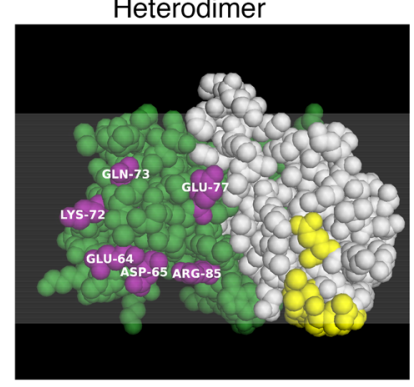

C

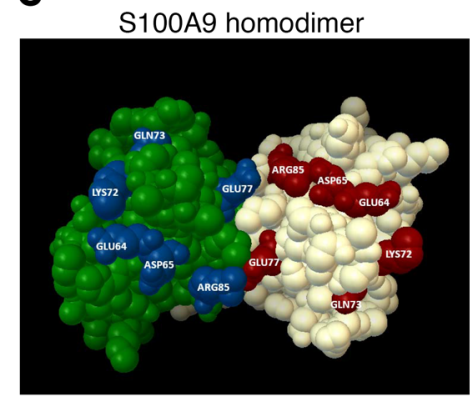

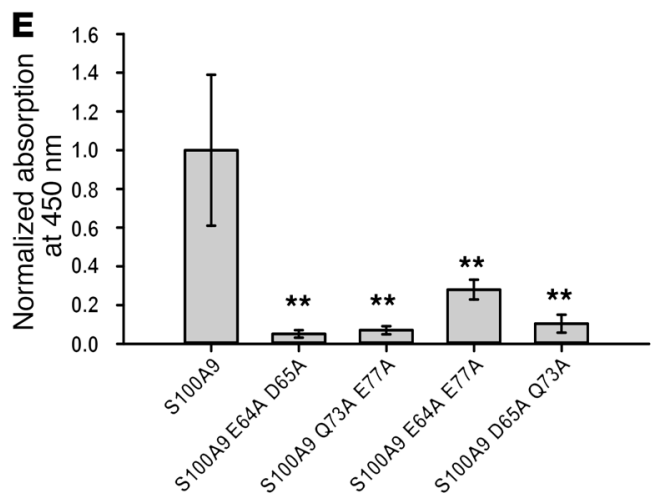

H
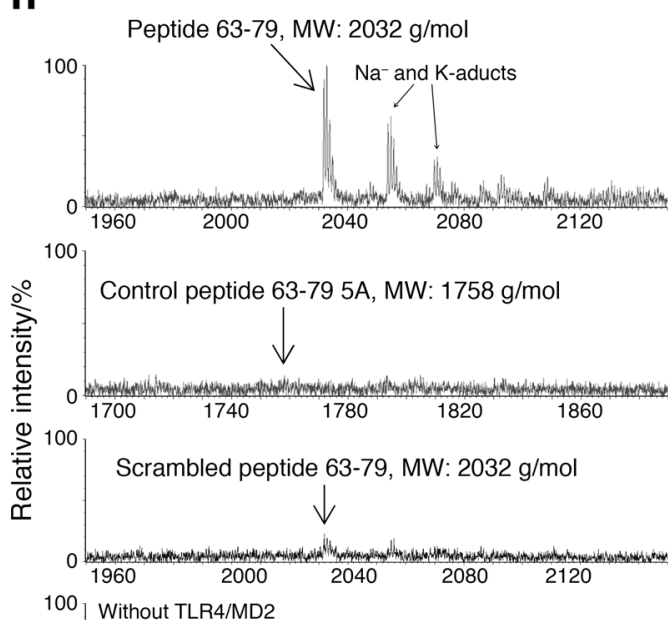

100 Without TLR4/MD2

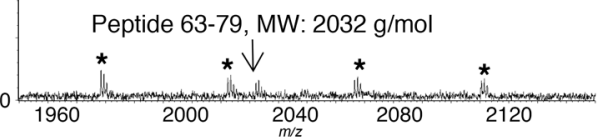

Figure 5. Identification of the S100A9-binding site on TLR4/MD2. (A-C) The PDB files of the S100A8/S100A9 tetramer (1XK4, A) and S100A9 dimer $(1 / R), C$ ) were retrieved from the RSCB PDB website. (B) 1XK4 was modified containing only the E (S100A8, gray) and G (S100A9, green) chains resembling 1 heterodimer. Masked aa in the tetramer were analyzed by Cluspro. (B, yellow- or cyan-labeled aa for S100A8 or S100A9). Labeled S100A9 aa were chosen for mutation studies (C). (D) Single mutated S100A9 was analyzed for TLR4/MD2 binding compared with WT S100A9. All S100A9 mutants showed reduced binding to TLR4/MD2. (E) Double-mutated S100A9 was analyzed for binding to TLR4/MD2 as shown in $\mathbf{D}$ and showed significantly reduced binding properties compared with WT S100A9. Data represent mean \pm SD of 5 independent experiments. ${ }^{*} P<0.05 ;{ }^{* *} P<0.01,1$-way ANOVA. (F) Monocytes were stimulated for 4 hours with intact S100A9 $(0 \mathrm{~h}=$ no trypsinization) or the fragment mixture, and TNF- $\alpha$ levels were quantified by ELISA. Western blot shows remaining intact S100A9 (insert). One representative of 3 independent experiments is shown. (C) Tryptic fragments of hS100A9 (F) were analyzed for TLR4/MD2 binding, and peptide 15 corresponding to aa 73-85 showed specific interaction with TLR4/MD2, as detected by nanoUPLC/ESI-Q-TOF MS/ MS ( $m / z$ 1614). Peptide 6 (NIETIINTFHQYSVK) was also detected in the control setting without TLR4/MD2 and reflects unspecific binding. (H) Peptides comprising aa 63-79 of S100A9 (MEDLDTNADKQLSFEEF, MW: $2032 \mathrm{~g} / \mathrm{mol}$ ), control peptides 63-79 5A (MAALDTNADAALSFAEF, MW: $1758 \mathrm{~g} / \mathrm{mol}$ ) or a scrambled peptide (DSLEMTEENLADQFKDF, MW: $2032 \mathrm{~g} / \mathrm{mol}$ ) were investigated for TLR4/MD2 binding as shown in G. Unspecific binding was analyzed by using peptides 63-79 without TLR4/MD2. Only S100A9 peptides 63-79 showed binding to TLR4/MD2 ( 3 independent experiments). Asterisks indicate polyethylene glycol impurities. 
A

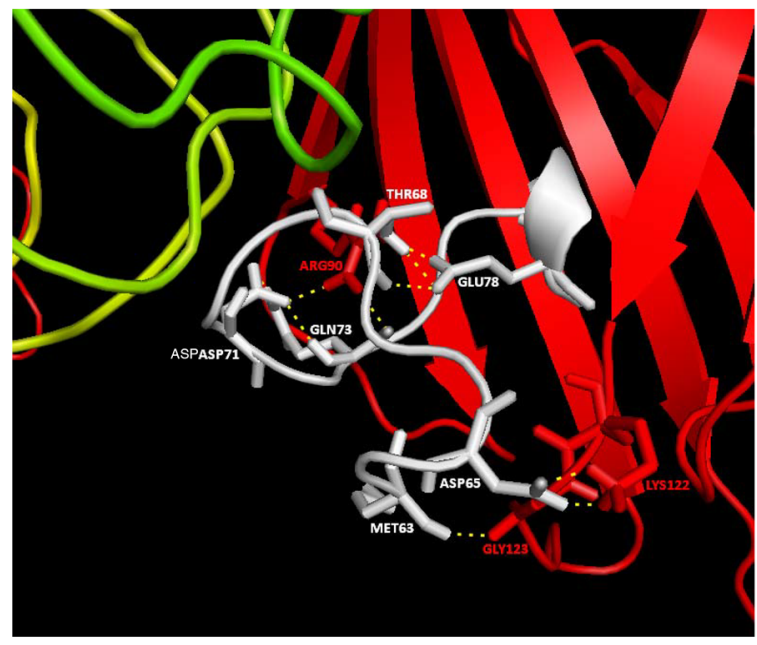

B

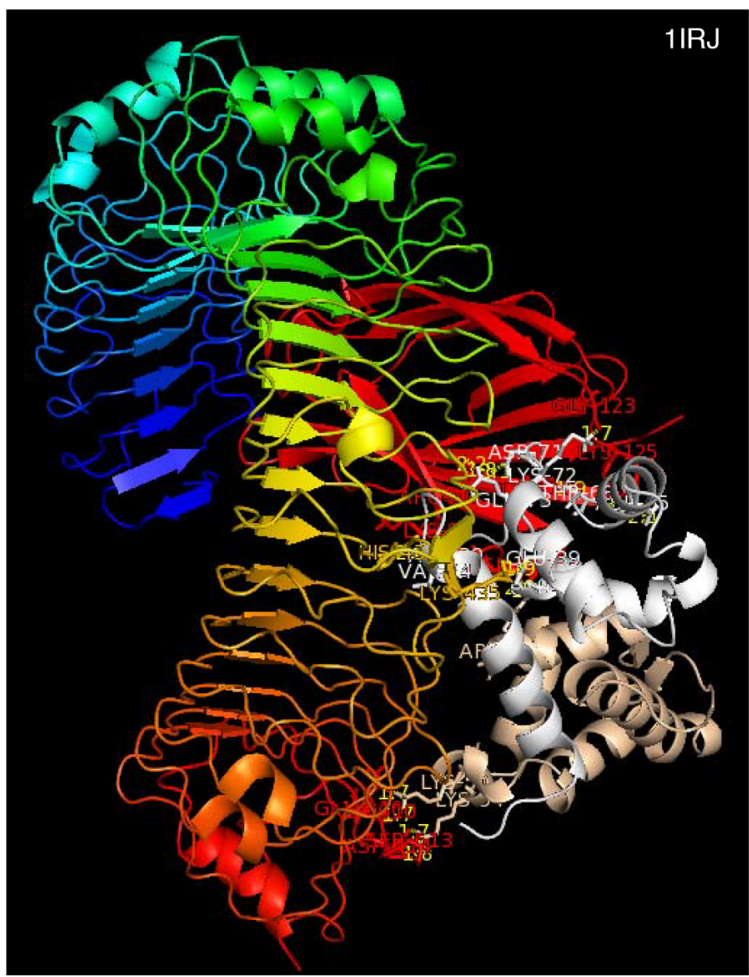

C

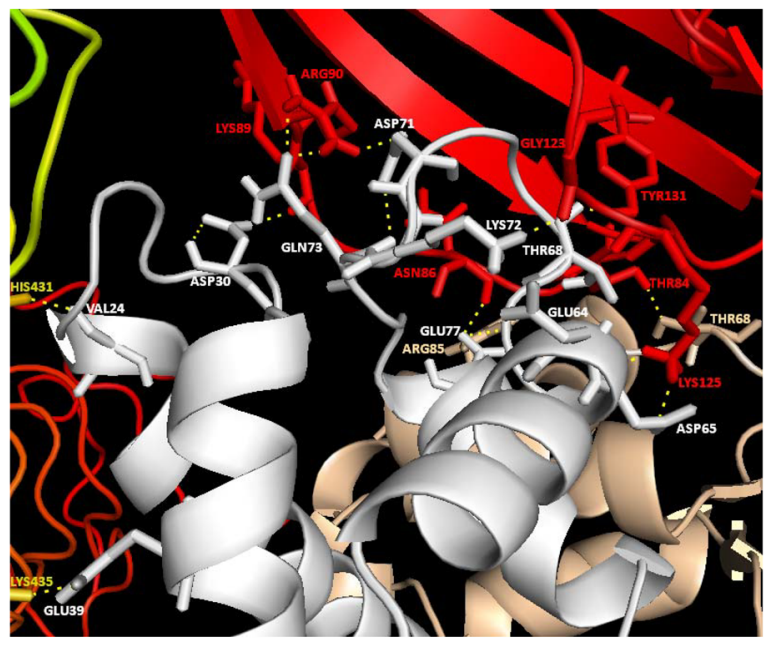

C

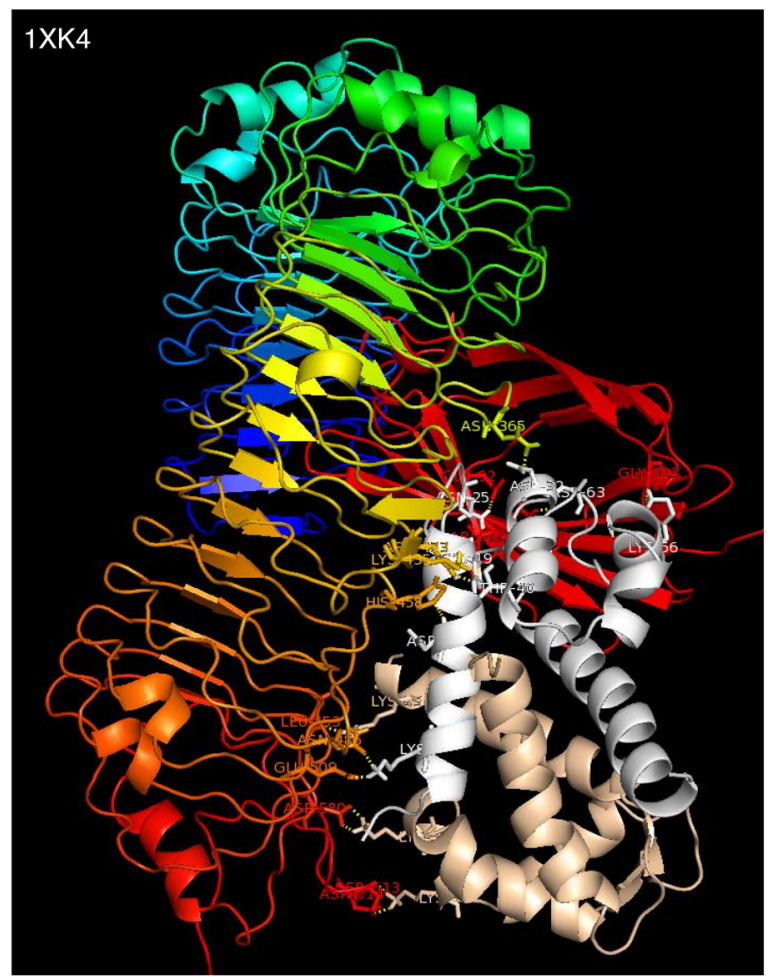

Figure 6. Proposed structural modeling of S100A9 homodimer interaction with TLR4/MD2. (A) Modeling of the S100A9 peptides 63-79 hTLR4/MD2 complex in ribbon representation showing a close-up view with the putative interaction aa (labeled in gray for S100A9 aa and red for MD2-specific aa) as indicated. The model is based on the crystal structure of the TLR4/MD complex (PDB ID 3FXI), with LPS being omitted and the coordinates of peptides 63-79 being taken from PDB ID: 1IRJ. (B) Model of the potential binding site of S100A9 homodimer and TLR4/MD2 showing a number of contact aa summarized in Table 1. (C) Close-up view of $\mathbf{B}$ focused on the S100A9 EF-hand II, MD2 binding region. (B and C) Model is based on the crystal structures of S100A9 (PDB ID: 1IRJ, chains $G$ and H) and of the human TLR4/MD2 complex (A chain of TLR4 and C chain of MD2) with LPS being omitted. The side chains of S100A9 aa are labeled in gray or brown and those of TLR4/MD2 in red. (D) Structural modeling of S100A8/S100A9 heterodimer (PDB ID 1XK4, chains E and G) interaction with TLR4/MD2 confirms identical binding regions for the homodimer S100A9 as well as for the heterodimer. Molecular modeling and graphics preparation were performed using Cluspro and PyMOL software packages.

experimental tool for mimicking S100A8/S100A9 dimer effects due to the fact that homodimers cannot tetramerize, but are of minor relevance in vivo $(35,37)$.

The identification of a specific molecular structure responsible for S100 TLR4/MD2 interaction presented here markedly increases the chances of addressing S100A8/S100A9 as a highly relevant target for therapeutic approaches. The heterocomplex, also known as calprotectin, is widely used as a biomarker for detection and monitoring the disease activity of inflammatory bowel disease, psoriasis, or rheumatoid arthritis (15). S100A8/ S100A9 expression predicts therapeutic efficiency and disease flares of arthritis, further emphasizing the functional significance 
of these proteins in clinically relevant diseases (38). The extraordinarily high expression of S100A8/S100A9 at local sites of active inflammation compared with clinically noninvolved tissue or systemic levels further highlights the relevance of the molecular mechanism described here for local inflammatory activity (30, 39). The frequently local and discontinuous patterns of inflammatory symptoms in many autoimmune diseases may be explained by the fact that local manifestation of clinical inflammation due to underlying systemic autoimmunity needs an additional tissuederived stress trigger such as locally active $\mathrm{S100}$ alarmins. The high local expression of these molecules during inflammation is also demonstrated in in vivo imaging studies of different inflammatory disease models in mice (27). General KO of the S100A8/ S100A9 system significantly blocks inflammation, and biological relevance of this system has been confirmed in various prelinical models of inflammatory processes, such as LPS-induced septic shock, arthritis, psoriasis, and SLE-like disease, all of which reflect the expression pattern of S100A8/S1000A9 in human diseases very well $(8,11,12,40)$. Finally, several sophisticated models of metastasis development during tumor progression indicate a role of S100 TLR4-driven inflammation in the establishment of premetastatic niches and the potential use of targeted blockade of S100A8/S100A9 to prevent dissemination of tumor cells $(41,42)$.

TLR4 has already been shown to play a role in sterile inflammation in the absence of any microbial ligands (1-4), and S100A8 and S100A9 are the major endogenous TLR4 ligands in many inflammatory diseases, especially rheumatoid arthritis, psoriasis, and inflammatory bowel disease $(38,40,43)$. TLR4-targeted therapies are currently under development for acute and chronic inflammation. In terms of therapy, targeting $\mathrm{S} 100$ proteins instead of TLR4 has an obvious advantage, as specific blocking of active S100A8/S100A9 heterodimers would not primarily affect the TLR4-dependent antimicrobial defense mechanisms. Although concentrations of S100A8/S100A9 are relatively high during inflammatory diseases, our data indicate that the pharmacologically relevant target is only the minor fraction of active heterodimers. There are already 2 lines of evidence that blocking of S100 proteins may be effective. Molecules of the so-called Q-compound family have been shown to inhibit the interaction of S100A8/ S100A9 with TLR4, show an antiinflammatory effect, and have been successfully used in phase III trials for treatment of multiple sclerosis $(44,45)$. In addition, recombinant peptide bodies have been shown to block S100-dependent effects in a murine model of tumor metastasis. However, it is not clear whether Q-compounds or these peptide bodies specifically address the TLR4-binding region of S100 proteins described here (46). Along with the mechanism of locally restricted activity of S100A8/S100A9-driven inflammation shown here, these molecules are promising candidates for a very specific and local antiinflammatory therapy avoiding systemic side effects. Taking our data together, we describe a molecular mechanism by which alarmins drive sterile inflammation restricted to local sites of injury by autoinhibitory regulation.

\section{Methods}

Animalstudies. The WT C57BL/6, S100A9-/-, TTP ${ }^{-/-}, \mathrm{TNF}^{-\alpha^{-/}}$, hTNF-tg (strain Tg197), and ihTNF-tg mouse strains have been described elsewhere $(13,18,26)$. All mouse lines had a C57BL/6 genetic back- ground except the ihTNF-tg mice, which consisted of C57BL/6 $\times$ FVB-N hybrids. For analysis of the role of S100A8/S100A9 proteins in the development of TNF- $\alpha$-mediated disorders, further hybrid mice with the S100A9 deficiency in combination with other KO genes and transgenes were generated and are described in Results. Genotyping was performed by PCR using primers and conditions as described elsewhere $(13,18,26)$.

Scoring criteria of the psoriasis-like skin disease of $\mathrm{TTP}^{-/-} \mathrm{S1OOA9^{-/- }}$ mice. To evaluate the severity of the psoriasis-like phenotype of TTP ${ }^{-}$ /- S100A9 ${ }^{-/-}$mice, an adapted PASI score was used, as follows: grade 0, $0 \%$ involved area; grade 1 , less than $10 \%$ involved area; grade 2 , $10 \%-29 \%$ involved area; grade 3, 30\%-49\% involved area; grade 4 , $50 \%-69 \%$ involved area; grade $5,70 \%-89 \%$ involved area; and grade 6, 90\%-100\% involved area.

Scoring criteria of ihTNF-tg/S1OOA9-- and ihTNF-tg mice. To induce expression of the human TNF- $\alpha$ transgene, 6-week-old mice were kept on drinking water containing dox $(1 \mathrm{mg} / \mathrm{ml}$, SigmaAldrich) and 5\% sucrose for the indicated times. The water was protected from light and exchanged every 3 days. Clinical assessments, such as body weight, paw swelling, and grip strength, were performed twice a week during the dox stimulation of the mice. Paw swelling was graded from 0 to 3 , and grip strength was graded from 0 to -3 as previously described (47).

Anti-TNF- $\alpha$ treatment. To neutralize the excess of TNF- $\alpha$ in $\mathrm{TTP}^{-/-} \mathrm{S} 100 \mathrm{A9}^{-/-}$mice, the animals received either $40 \mathrm{mg} / \mathrm{kg}$ certolizumab (UCB), a PEGylated Fab' fragment of a humanized TNF- $\alpha$ inhibitor monoclonal antibody, or a monoclonal antibody of irrelevant specificity as control antibody into the back skin on days 4,6 , and 8 after birth. The antibody is already in clinical use as arthritis and psoriasis therapy.

Reagents. LPS from E. coli (055:B5) was purchased from SigmaAldrich. SB202190 and PD98059, specific inhibitors for p38 MAPK and MEK were from Calbiochem. Staurosporine and GF109203X (inhibitors of protein kinase C) were from Alexis Biochemicals and Molecular Probes (via MoBiTec). The TLR4 antagonist RS-LPS (LPS from Rhodobacter sphaeroides) was from Invivogen, and the blocking mouse monoclonal antibody to human TLR4 (HTA125) was from Abcam.

Determination of cytokine concentrations. TNF- $\alpha$ and IL-1 $\beta$ protein levels were analyzed in culture supernatants using commercial ELISA kits (BD Bioscience). Cytokine concentrations in tissues were assayed using the FACS-based FlowCytomix Multiplex Kit (eBioscience).

Cells and cell-culture conditions. Methods of cell purification and culture conditions have been described in detail elsewhere (8). Briefly, for stimulation experiments, human monocytes $\left(10^{6}\right.$ cells $\left./ \mathrm{ml}\right)$ were treated with varying concentrations $(100 \mathrm{ng} / \mathrm{ml}$ to $5 \mu \mathrm{g} / \mathrm{ml})$ of purified S100A8, S100A9, or S100A8/S100A9 proteins or mutants thereof, LPS $(1-10 \mathrm{ng} / \mathrm{ml})$, and/or IFN $-\gamma(500 \mathrm{U} / \mathrm{ml})$ for the times indicated in figure legends.

Human monocytes were isolated from blood samples after leukapheresis via Pancoll (Pan Biotech) and subsequent Percoll (GE Healthcare) gradient centrifugation (48). Cells were cultivated overnight in Teflon bags in McCoy's 5a Medium (Biochrome) supplemented with 15\% FCS (Gibco Life Technologies, Thermo Fisher Scientific) prior to use for stimulation or in functional assays.

Mouse BMCs were flushed from femurs and tibias with Dulbecco's medium. Erythrocytes were lysed with lysis buffer $\left(155 \mathrm{mM} \mathrm{NH}_{4} \mathrm{Cl}, 10\right.$ $\mathrm{mM} \mathrm{KHCO}, 0.1 \mathrm{mM}$ EDTA) for 5 minutes at room temperature, and 
the remaining cells were washed once with PBS. Murine whole-blood cells were collected from heparin blood after lysis of erythrocytes with lysis buffer. Collected cells were either lysed for Western blot analysis or centrifuged on glass slides for S100A8 and S100A9 staining.

A549 human alveolar epithelial cells (ATCC, CCL-185) were grown in HAM's F-12 medium supplemented with $2 \mathrm{mM}$ L-glutamine and $10 \%$ heat-inactivated FCS. The experiments reported here were performed using confluent cells in serum-rich medium.

HEK293 cells stably transfected with human TLR4, CD14, and MD2 genes or TLR4 only as well as control cells transfected with empty control vectors were purchased from Invivogen. HEK293 cells stably transfected with human receptor for advanced glycation end products (RAGE) were provided by Marco E. Bianchi (San Raffaele Scientific Institute, San Raffaele University, Milan, Italy). HEK293 cells were grown in DMEM with $4.5 \mathrm{~g} / \mathrm{l}$ glucose, $10 \% \mathrm{FBS}$ at $37^{\circ} \mathrm{C}$ in $5 \% \mathrm{CO}_{2}$.

Primary epidermal keratinocytes were isolated from the skin of 2- to 3-day-old newborn mice as described (49) and cultured in FAD medium containing $50 \mu \mathrm{M}$ calcium ions. All cell culture media (Merck Millipore) and all other reagents were entirely free of endotoxin, as checked by limulus amebocyte lysate (LAL) assay (BioWhittaker).

Antibody labeling. Rabbit-derived antibodies addressing S100A8 were purified via protein G-sepharose and labeled with the fluorochrome Cy5.5 according to the manufacturer's instructions (GE Healthcare). Briefly, $5 \mathrm{mg}$ of the antibody was dialyzed toward $100 \mathrm{mM} \mathrm{Na}_{2} \mathrm{CO}_{3}$ buffer, $\mathrm{pH}$ 8.0, and a 20-fold excess of the fluorochrome was added for 90 minutes at room temperature. The resulting tracer was purified from unbound dye using SEC (PD10 column). The labeling efficacy (dye/ antibody ratio) was determined on the basis of UV-spectra of the purified dye-antibody compound using PBS as a reference buffer. Typically, the labeling resulted in 2.5-3.0 fluorochrome molecules per antibody.

FRI. FRI was performed using the In-Vivo FX PRO Imaging Station (Bruker Biospin MRI GmbH). For imaging of Cy5.5-labeled S100A8 antibody, the excitation light was set to $630 \mathrm{~nm}$ using an appropriate bandpass filter. Emission at $700 \mathrm{~nm}$ was recorded using a filter-equipped high-sensitivity (4-million-pixel) cooled chargecoupled device (CCD) camera. Acquisition time was 5 seconds for each image, followed by a photography-style white light image for the biodistribution, respectively. Mice were held under isoflurane inhalation anesthesia (2.0\% isoflurane in air) for the duration of the scan. As the total scan time was usually less than 1 minute, physiological effects due to a significant decrease of body temperature were not to be expected. Mice were intravenously injected with Cy5.5-labeled S100A8 antibody ( $\alpha$-S100A8-Cy5.5, 2 nmol of Cy5.5 100 $\mu$ g antibody in total) 24 hours prior to OI.

Protein expression and purification of $100 \mathrm{WT}$ and mutant proteins. Recombinant S100 proteins were expressed in E. coli BL21 (DE3) cells and purified as previously described $(17,35)$. Briefly, bacteria cells were transformed with pET11/20 expression vectors containing the cDNAs for murine or human WT S100A8 or WT S100A9 or the human S100A9 mutants N69A, E78A, E64A, D65A, K72A, Q73A, E77A, R85A, E64A+D65A, Q73A+E77A, E64A+E77A, and D65A+Q73A; after growing at $37^{\circ} \mathrm{C}$ in $2 \times$ yeast tryptone medium for 24 hours, cells were harvested and lysed. The inclusion bodies (IB) were pelleted and dissolved in $8 \mathrm{M}$ urea buffer. To establish proper refolding, samples were first adjusted to $\mathrm{pH}$ 2.0-2.5 by adding hydrochloric acid, and after 60 minutes incubation at room temperature, proteins were stepwise dialyzed against Tris buffers in the presence of $2 \mathrm{mM}$ DTT to become adapted to $\mathrm{pH}$ 7.4. After centrifugation (10 minutes, $60,000 \mathrm{~g}, 4^{\circ} \mathrm{C}$ ) to pellet the aggregated material, samples were further dialyzed against appropriate buffers for anion exchange column and gel filtration chromatography. The protein concentrations were determined by UV absorption at $280 \mathrm{~nm}$ using a specific absorption coefficient of 1.02 (humS100A8), 0.53 (humS100A9 and mutants), 0.76 (humS100A8/ S100A9 and mutants) 0.40 (murS100A8), 0.11 (murS100A9), or 0.25 (murS100A8/S100A9) $\mathrm{ml} / \mathrm{mg}^{-1} / \mathrm{cm}^{-1}$, respectively. Proper complex formation of S100A8/S100A9 and mutants thereof was enabled by including a denaturation/renaturation step. Both $\mathrm{S} 100$ subunits were mixed in an equimolar ratio. Samples were adjusted to $\mathrm{pH} 2.0-2.5$ by adding hydrochloric acid in $0.1 \mathrm{M}$ glycine buffer. After 60 minutes incubation at room temperature, samples were dialyzed stepwise to become adapted to $20 \mathrm{mM}$ Tris (pH 8.5), 1 mM EDTA, 1 mM EGTA, and $2 \mathrm{mM}$ DTT, pH 8.5, for proper refolding and complex formation. After centrifugation (10 minutes, $60,000 \mathrm{~g}, 4^{\circ} \mathrm{C}$ ) to pellet the aggregated material, samples were applied to anion exchange column chromatography. Only fractions that contained equal amounts of both proteins, as detected by SDS-PAGE, were collected. Samples were stored as stock solutions at $-20^{\circ} \mathrm{C}$. Correct refolding and complex formation were assessed by SDS-PAGE, MALDI-mass spectrometry (MALDIMS), and electrospray ionization-MS (ESI-MS) $(16,50)$. To confirm the structural integrity of double-mutated S100A9 homodimers, fluorescence spectra were recorded at $20^{\circ} \mathrm{C}$ with a spectrofluorometer (Spex, Model FluoroMax II, Instruments SA) using protein concentrations of $5 \mu \mathrm{g} / \mathrm{ml}$. Changes in the conformation of S100A9 due to the mutation or mutations would be easily detectable by altered intrinsic Trp and Tyr fluorescence properties. All fluorescence spectra were buffer corrected (35). Possible endotoxin contaminations were excluded by LAL assay (BioWhitaker). LPS could not be detected or was lower than 1 $\mathrm{pg} / \mu \mathrm{g}$ S100 protein in the different batches. As an additional control, S100A9 protein samples were completely heat inactivated at temperatures at which LPS was still fully active, but no biological response was registered. Moreover, LPS contaminations were further excluded by blocking experiments using polymyxinB sulfate (Sigma-Aldrich) for all preparations.

DNA microarray hybridization. Total cellular RNA (MachereyNagel) was isolated from human monocytes that were exposed to $5 \mu \mathrm{g} / \mathrm{ml}$ of S100A8, S100A9, or S100A8/S100A9 or $1 \mathrm{ng} / \mathrm{ml} \mathrm{LPS} \mathrm{or}$ to medium for 4 hours ( 3 independent experiments) and used for analysis by Illumina human HT-12 Expression BeadChips. The protocol was performed according to the manufacturer's instructions. Data were imported into Partek Genomics Suite V6.6 (PGS) (Partek) using robust multiarray average (RMA), which is an algorithm used for background correction, $\log _{2}$ transformation, and quantile normalization of expression data prior to batch correction. A 1-way ANOVA model was performed to calculate the 1,000 most variable and differentially expressed genes between the different stimulating conditions. Differentially expressed genes were defined by a fold change (FC) greater than 2.5 or less than -2.5 and a false discovery rate-corrected (FDRcorrected) $P$ value of less than 0.05 . To visualize the structure within the data, we performed hierarchical clustering (HC) on the 1,000 most variable genes, with default settings in PGS, on FDR-corrected $P$ values according to the expression values of the samples across the conditions as described (9).

Quantitative real-time PCR. RNA from HEK cells, human monocytes, or snap-frozen mouse tissue was isolated using the NucleoSpin 
RNA II Kit (Macherey-Nagel), and $1 \mu \mathrm{g}$ of total RNA was reversetranscribed to cDNA using RevertAid H Minus Transcriptase (Fermentas). Expression levels of selected genes were confirmed by quantitative reverse-transcriptase PCR (RT-PCR), which was performed using Sybr Green (PeqLab). Data were acquired with the CFX 384 Real-Time System and CFX-manager software, version 3.0 (Bio-Rad). Each measurement was set up in duplicate, and the relative expression was calculated as $2^{-\triangle \Delta C t}$ using ribosomal protein L (RPL), GAPDH, or $\beta$-actin as the housekeeping control genes. Sample data are presented as fold induction compared with naive cells.

The following primers were used for human PCR analysis: IL-6 forward, 5'-AGAGGCACTGGCAGAAAACAAC-3'; IL-6 reverse, 5'-AGGCAAGTCTCCTCATTAATCC-3'; SOCS1 forward, 5'-AGCTCCTTCCCCTTCCAGATTT-3'; SOCS1 reverse, 5'-CCACATGGTTCCAGGCAAGTAATA-3'; SOCS3 forward, 5'-CCTCAAGACCTTCAGCTCCAA-3'; SOCS3 reverse, 5' -ACTGCGCTCCAGTAGAAGCC-3';TNF-AIP3forward,5'-CGTCAGAAGCTGGCTGATTTG-3'; TNF-AIP3 reverse, 5'-CCTTGTCGGTCTCCTCCATTTC-3'; IL-10 forward, 5'-GCTGAGAACCAAGACCCAGACA-3'; IL-10 reverse, 5'-CGGCCTTGCTCTTGTTTTCA-3'; IL-1 $\alpha$ forward, 5 '-AGACCTACGCCTGGTTTTCC-3'; IL-1 $\alpha$ reverse, 5 '-GCTGAAGGAGATGCCTGAGAT-3'; CD80 forward; 5'-CTGCTTTGCCCCAAGATGC-3'; CD80 reverse, 5'-CAGATCTTTTCAGCCCCTTGC-3'; SOCS2 forward, 5'-CTGACCAAACCGCTCTACACGT-3'; SOCS2 reverse, 5'-TTGTTGGTAAAGGCAGTCCCC-3'; NF-кB forward, 5'-TTATGTATGTGAAGGCCCATCC-3'; NF- $\mathrm{B}$ reverse, 5'-TGGTCCCACATAGTTGCAGATT- $3^{\prime}$; TNF- $\alpha$ forward, 5'-CTTCTCGAACCCCGAGTGAC-3'; TNF- $\alpha$ reverse, $5^{\prime}$-TGAGGTACAGGCCCTCTGATG-3'; RAGE forward; 5'-CTACCGAGTCCGTGTCTACCA-3'; RAGE reverse, 5'-CATCCAAGTGCCAGCTAAGAG-3'; TLR-4 forward, 5'-CCAGAACCAAACGATGGACT-3'; TLR-4 reverse, 5'-CCTGTCCCTGAACCCTATGA-3'; CXCL10/IP-10 forward, 5'-AGACATCTCTTCTCACCCTTC-3'; CXCL10/IP-10 reverse, 5'-GGAACCTCCAGTCTCAGCACCA-3'; CCL5 forward, 5'-CAGTGGCAAGTGCTCCAACC-3'; CCL5 reverse, 5'-CCATCCTAGCTCATCTCCAAAGAGT-3'; RPL forward, 5'-AGGTATGCTGCCCCACAAAAC-3'; RPL reverse, 5'-TGTAGGCTTCAGACGCACGAC-3'; GAPDH forward, 5'-CAAATTCCATGGCACCGTCA-3';

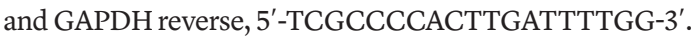

The following primers were used for murine PCR analysis: TNF- $\alpha$ forward, 5'-AGAAACACAAGATGCTGGGACAGT-3'; TNF- $\alpha$ reverse, 5 -CCTTTGCAGAACTCAGGAATGG-3'; IL-1 $\beta$ forward, 5'-TGGGCTGGACTGTTTCTAATGC-3'; IL-1 $\beta$ reverse, 5'-TGTCTTGGCCGAGGACTAAGG-3'; IL-6 forward, 5'-TGAGATCTACTCGGCAAACCTAGTG-3'; IL-6 reverse, 5'-CTTCGTAGAGAACAACATAAGTCAGATACC-3'; IL-17A forward, 5'-CAGAAGGCCCTCAGACTACC-3';IL-17Areverse,5'-TCTCGACCCTGAAAGTGAAG-3'; IL-22 forward, 5'-ACCTTTCCTGACCAAACTCA-3'; IL-22 reverse, 5'-AGCTTCTTCTCGCTCAGACG-3'; IL-23 forward, 5'-ATCCAGTGTGAAGATGGTTGTGA-3'; IL-23 reverse, 5'-CTGGAGGAGTTGGCTGAGTC-3'; IL-36 forward 5'-TACATGGGAGTGCAAAGGCC-3'; IL-36 reverse 5'-AGTGGGCAGCTCCCTTTAGAGC-3'; IFN- $\gamma$ forward 5'-TGCTGATGGGAGGAGATGTCTAC-3'; IFN- $\gamma$ reverse 5'-TTTCTTTCAGGGACAGCCTGTTAC-3'; RPL forward, 5'-TGGTCCCTGCTGCTCTCAAG-3'; RPL reverse, 5'-GGCCTTTTCCTTCCGTTTCTC-3'; GAPDH forward, 5'-CCCACTCTTCCACCTTCGATG-3'; GAPDH reverse, 5'-GTCCACCACCCTGTTGCTGTAG-3'; $\beta$-actin forward, 5'-AAGGAGA-
AGCTGTGCTACGTC-3'; and $\boldsymbol{\beta}$-actin reverse, 5'-AACCGCTCATTGCCAATGGTG-3'.

Histology and immunohistochemistry. The paws were fixed overnight in $4 \%$ paraformaldehyde, decalcified with $0.5 \mathrm{M}$ EDTA solution, and embedded in paraffin. Sections measuring $4 \mu \mathrm{m}$ in thickness were analyzed. The extent of inflammation and cartilage damage in the paws was determined by morphometry as described elsewhere (47). Usually, 4 different sections per mouse sample were quantified from 5 to 6 mice for each mouse line. Proteoglycan loss was determined as the percentage of destained cartilage area. For skin samples, mice were sacrificed at day 9, and skin from the back was fixed in $4 \%$ paraformaldehyde or embedded in Tissue-Tek (Sakura) for cryosectioning. Fixed skin samples were embedded in paraffin according to procedures used for H\&E staining and immunohistochemistry. For immunohistochemistry, paraffin skin sections $(4 \mu \mathrm{m})$ were dewaxed, blocked 30 minutes with $10 \%$ normal goat serum (Invitrogen), and incubated with rabbit anti-S100A9 or antiS100A8 antibodies for 1 hour at room temperature. Vectastain ABC-AP (BA-1000) and Vector Red Substance Kits (SK-5100) (Vector Laboratories) were used for visualization of the stained proteins. For immunofluorescence analyses, sections $(5 \mu \mathrm{m})$ of frozen tissue were fixed in ice-cold acetone. After blocking with $10 \%$ normal goat serum in PBS for 30 minutes, the sections were washed in PBS $(3 \times 5$ minutes $)$. Primary antibodies dissolved in PBS were applied for 1 hour at room temperature or overnight at $4^{\circ} \mathrm{C}$. After washing in PBS, sections were incubated with anti-rabbit or anti-rat Alexa Fluor 488-coupled IgG. Sections were then counterstained with propidium iodide or DAPI. Finally, after washing in PBS, sections were fixed with $4 \%$ paraformaldehyde and mounted in Mowiol (Sigma-Aldrich). The following primary polyclonal antibodies were used: anti-keratin 14 (1:100; clone poly 19053, BioLegend), antiK10 (1:100; clone poly 19054, BioLegend), anti-Lor (1:2000; clone poly 19051, BioLegend) (Covance). Anti-Ki67 (1:100, ab15580, Abcam) rat monoclonal antibodies were used on frozen sections as follows: F4/80 (1:100; clone Cl:A3-1, Bio-Rad), anti-Gr-1 (1:20; clone RB6-8C5, BD Bioscience), and anti-CD3 (1:1000; clone 17A2, Thermo Fisher). Sections were counterstained with propidium iodide to visualize the nuclei. Fluorescence stainings were analyzed using a Zeiss Axio Vision Microscope.

Detection of MyD88 translocation by immunofluorescence microscopy. A549 cells were cultured on fibronectin-coated LabTec chamber slides (Nunc, Thermo Scientific) until confluence. After stimulation, as indicated in the figure legends, cells were fixed with formaldehyde $\left(3.7 \%, 20^{\circ} \mathrm{C}, 10\right.$ minutes $)$ and permeabilized with Triton-X $100(0.5 \%$, $20^{\circ} \mathrm{C}, 10$ minutes). After blocking of unspecific binding sites with FCS $\left(10 \%, 4^{\circ} \mathrm{C}\right.$, overnight), samples were processed for single-labeling immunofluorescence using a polyclonal rabbit antibody to human MyD88 (ALX-210-900, Alexis Biochemicals) (51).

Immunoprecipitation of IRAK- 1 . Human monocytes $\left(20 \times 10^{6}\right.$ cells per sample) were preincubated overnight with human recombinant IFN- $\gamma$ (500 U/ml, Bender MedSystems) or with medium alone and subsequently stimulated with $10 \mathrm{ng} / \mathrm{ml}$ LPS or S100A9 $(5 \mu \mathrm{g} / \mathrm{ml})$ for 45 minutes. Cells were then lysed in $1 \mathrm{ml}$ lysis buffer (0.5\% Nonidet P-40, 10\% glycerol, 50 mM HEPES, pH 7.9, $250 \mathrm{mM} \mathrm{NaCl}, 20 \mathrm{mM}$ glycerophosphate, $5 \mathrm{mM}$ p-nitrophenyl phosphate, $1 \mathrm{mM}$ EDTA, $1 \mathrm{mM}$ sodium orthovanadate, 5 $\mathrm{mM}$ dithioerythritol, $1 \times$ complete protease inhibitors; Roche). IRAK-1 was immunoprecipitated using $2 \mu \mathrm{g}$ per sample IRAK-1-specific monoclonal antibody (catalog sc-5288, Santa Cruz Biotechnology Inc.) and a mixture of protein $\mathrm{G}$-sepharose and protein A-agarose (Roche). In vitro kinase assays were performed as described previously (52). 
Western blot analysis. Nuclear cell extracts were prepared using the extraction kit (Epigentek). For each stimulus, equal cytosolic amounts of monocytes (p-IRAK-1, catalog sc-130197, Santa Cruz Biotechnology Inc.), keratinocytes (murS100A8, homemade), BMCs (murS100A8), or nuclear extracts of monocytes (P50 [ab7971], P65 [ab7970] Abcam) were separated on SDS-polyacrylamide gels and transferred to nitrocellulose membranes (Schleicher \& Schuell BioScience). Peptidic fragments of S100A9 generated by trypsin digestion were separated on SDS-polyacrylamide gels and transferred to nitrocellulose membranes, which were then blocked with $5 \%$ skim milk powder and subsequently probed with primary antibodies overnight at $4^{\circ} \mathrm{C}$. Afterwards, bound primary antibody was detected with HRP-conjugated secondary antibodies and developed with enhanced chemiluminescence system (ECL).

Proteasome and ubiquitin enzymes E1 and E2 inhibition assays. BMCs were isolated from $\mathrm{S} 100 \mathrm{~A} 9^{-/-}$mice. Cell pellet was resuspended in BMC growth medium (DMEM, 10\% FCS, 20\% L929 cell supernatant, $1 \%$ penicillin/streptomycin, $2 \%$ L-glutamine), and cells were seeded at $1 \times 10^{6}$ cells/well in a 24 -well plate at $1 \mathrm{ml}$ per well. Cells were then treated with inhibitors of the proteasome (MG132, UBPBio), ubiquitin-activating enzyme E1 (PYR-41, UBPBio), or ubiquitinconjugating enzyme E2 (BAY-11-7821, Tocris Bioscience) at the indicated time points and concentrations and incubated at $37^{\circ} \mathrm{C}, 5 \% \mathrm{CO}_{2}$. The control cells were treated with DMSO.

Tryptic digestion of human S100A9 homodimers. Immobilized TPCK trypsin ( $25 \mu \mathrm{l}$ of settled gel, Pierce) was used to digest $30 \mu \mathrm{g}$ human $\mathrm{S100 \textrm {A } 9}$ at $37^{\circ} \mathrm{C}$ for different time points, as indicated in Figure $5 \mathrm{~F}$. Subsequently, samples were centrifuged ( 5 minutes, $400 \mathrm{~g}$ ) using a resin separator to remove trypsin-loaded beads. Aliquots of the supernatants were taken either for analyses by SDS-PAGE/Western blotting or for stimulation of human monocytes for 4 hours. TNF- $\alpha$ concentrations in supernatants of stimulated monocytes were then determined by ELISA (OptEIA, BD Biosciences). The fragments generated by tryptic digestion of hS100A9 were incubated with TLR4/ MD2-coupled protein A/G agarose beads in the presence of $100 \mu \mathrm{M}$ $\mathrm{Ca}^{2+}$ ions. Bound fragments were eluted at $\mathrm{pH} 2.0$ and analyzed via MALDI-TOF and LC-coupled tandem mass spectrometry.

Immunoprecipitation studies to identify TLR4/MD2-binding peptides. Anti-His antibody (catalog 37-2900, Invitrogen) and His-tagged TLR4/MD2 (R\&D Systems) were mixed and coupled to protein A/G agarose (Pierce, Thermo Scientific). Tryptic peptides of S100A9 were added in the presence of $1 \mathrm{mM}$ calcium. Bound peptidic fragments were eluted at $\mathrm{pH} 2.0$ and analyzed by nano-ESI-Q-TOF and MALDITOF mass spectrometry. Identical experiments were performed to analyze the binding of the chemically synthesized peptides of S100A9 (aa 63-79, MEDLDTNADKQLSFEEF), corresponding control peptides (aa 63-79 5A, MAALDTNADAALSFAEF, aa 63-79 2A, MEALDTNADKALSFEEF, aa 63-79 2A, MADLDTNADKQLSFAEF; bold letters indicate introduced mutations), or a scrambled peptide of S100A9 (aa DSLEMTEENLADQFKDF).

Binding of WT S100A9 and mutated S100A9 to TLR4/MD2. Binding of S100A9 to TLR4/MD2 was analyzed by a modified S100 ELISA. Briefly, TLR4/MD2 (R\&D Systems) was coupled to the wells of a plate and served as a capturing molecule. S100A9 or mutant S100A9 proteins were added at a concentration of $2 \mu \mathrm{g} / \mathrm{ml}$ each in the presence or absence of $100 \mu \mathrm{M}$ calcium. Bound S100 proteins were detected by the addition of a primary anti-S100A9 antibody $(1 \mu \mathrm{g} / \mathrm{ml}$, polyclonal rabbit, homemade) and the secondary anti- rabbit IgG antibody coupled to HRP (1 $\mu \mathrm{g} / \mathrm{ml}$, Cell Signalling Technology). Tetramethylbenzidine (TMB) was used as substrate to quantify binding efficiency by absorbance readings at $450 \mathrm{~nm}$ (Anthos Mikrosysteme).

Computer-assisted ligand/receptorinteraction studies. PDB files of an S100A8/S100A9 tetramer (PDB ID: 1XK4, chains E,F,G,H), S100A9 tetramer (PDB ID: 1IRJ), and TLR4/MD2 dimer (PDB ID: 3FXI) were retrieved from the RSCB PDB website. The S100A8/S100A9 PDB file was modified so that it contained only the $\mathrm{E}$ and $\mathrm{G}$ chains resembling the heterodimer. The S100A9 PDB file was modified so that it contained only the $\mathrm{G}$ and $\mathrm{H}$ chains resembling 1 homodimer. The TLR4/ MD2 PDB file was modified so that it contained only the A chain of TLR4 and C chain of MD2. The modified PDB files of S100 proteins were then docked against TLR4/MD2 using Cluspro (http://cluspro. bu.edu/login.php) (53). PyMOL (https://pymol.org/2/) and Swiss$\mathrm{Pdb}$ viewer (https://spdbv.vital-it.ch/) software packages were used to visualize the docking interactions. We concentrated our analyses on the identification of aa that are not involved in $\mathrm{Ca}^{2+}$ binding and are sterically free for binding to TLR4. The aa in S100A9 (positions 64, 65, $72,73,77$, and 85) were chosen for mutation studies.

SEC. SEC experiments were performed using a Superdex 75 loaded column connected with an Äktapurifier 900 FPLC (GE Healthcare) controlled by UNICORN software. The SEC separation runs were performed on a $5.0 \times 640 \mathrm{~mm}$ column, and UV detection was conducted at a wavelength of $280 \mathrm{~nm}$ by running a linear flow rate of $0.25 \mathrm{ml} /$ min. The column was calibrated with $8.56 \mathrm{kDa}$ ubiquitin, $12.4 \mathrm{kDa}$ cytochrome $c, 23.3 \mathrm{kDa}$ trypsin, $43 \mathrm{kDa}$ ovalbumin, and $66 \mathrm{kDa}$ serum albumin, and the running buffer was either HBS, $1 \mathrm{mM}$ EDTA, $1 \mathrm{mM}$ DTT, pH 7.4. or HBS, $5 \mathrm{mM} \mathrm{Ca}^{2+}, 1 \mathrm{mM}$ DTT, pH 7.4.

Statistics. Values are shown as mean \pm SD unless otherwise stated. $P$ values are given in the figure legends, and values of $P>0.05$ were considered not to be significant. Statistical analyses were performed by parametric tests ( 2 tailed $t$ test or 1-way ANOVA) and the MannWhitney $U$ test.

Study approval. Experimental procedures were approved by the local ethics committee in accordance with German regulations of the Society for Laboratory Animal Science, the European Health Law of the Federation of Laboratory Animal Science Associations, and the Landesamt für Natur, Umwelt und Verbraucherschutz Nordrhein-Westfalen.

Data availability. All original microarray data were deposited in the NCBI's Gene Expression Omnibus database (GEO GSE109646). Further information about the data and about the reagents used is available upon request to the corresponding author.

\section{Author contributions}

T Vogl, AS, JR, SL, TL, and TP designed and supervised the study and the experiments, performed animal studies, and wrote the manuscript. VW, T Völler, ST, SZ, DC, KL, MF, PP, and CW performed animal studies and experiments and analyzed the data. SH, CG, and M Schäfers performed PET and OI experiments. SKJ, OP, M Stoll, and JLS performed the microarray study and analyzed the data. SK and AD performed mass spectrometry experiments. All authors edited the manuscript.

\section{Acknowledgments}

We thank H. Berheide, H. Hater, and D. Lagemann for excellent technical support. This work was supported by grants from 
Cluster-of-Excellence EXC 1003 Cells in Motion, Münster, Germany (FF project-2013-24 to JR), the Interdisciplinary Center of Clinical Research at the University of Muenster (Vo2/004/14, Ro2/003/15, Lo2/004/16 and core unit PIX to T Vogl, JR, and KL), the German Research Foundation (DFG) (CRC 1009 B7, B8, and B9 to KL, T Vogl, and JR), CRC Transregio-128 (A2 to JR and A10 to KL, CRC 656 A9 to T Vogl and SH), the Federal Ministry of Education and Research (BMBF), project AID-NET and E-RARE, TreatAID (to JR), and the CRC 704 and Excellence Cluster Immuno-
Sensation (to JLS). T Völler was supported by a DFG postdoctoral fellowship (grant VO 2273/1-1).

Address correspondence to: Thomas Vogl, Institute of Immunology, University of Münster, Röntgenstr. 21, 48149 Münster, Germany. Phone: 0049.2518352943; Email:vogl@uni-muenster.de.

T Völler's present address is: Institute of Microbiology, Eidgenössische Technische Hochschule (ETH) Zurich, Zurich, Switzerland.
1. Rock KL, Latz E, Ontiveros F, Kono H. The sterile inflammatory response. Annu Rev Immunol. 2010;28:321-342.

2. Nathan C, Ding A. Nonresolving inflammation. Cell. 2010;140(6):871-882.

3. Chen GY, Nuñez G. Sterile inflammation: sensing and reacting to damage. Nat Rev Immunol. 2010;10(12):826-837.

4. Oppenheim JJ, Tewary P, de la Rosa G, Yang D. Alarmins initiate host defense. Adv Exp Med Biol. 2007;601:185-194.

5. Harris HE, Andersson U, Pisetsky DS. HMGB1: a multifunctional alarmin driving autoimmune and inflammatory disease. Nat Rev Rheumatol. 2012;8(4):195-202.

6. Chan JK, et al. Alarmins: awaiting a clinical response. J Clin Invest. 2012;122(8):2711-2719.

7. Edgeworth J, Gorman M, Bennett R, Freemont $\mathrm{P}$, Hogg N. Identification of p8,14 as a highly abundant heterodimeric calcium binding protein complex of myeloid cells. J Biol Chem. 1991;266(12):7706-7713.

8. Vogl T, et al. Mrp8 and Mrp14 are endogenous activators of Toll-like receptor 4, promoting lethal, endotoxin-induced shock. Nat Med. 2007;13(9):1042-1049.

9. Fassl SK, et al. Transcriptome assessment reveals a dominant role for TLR4 in the activation of human monocytes by the alarmin MRP8. JImmunol. 2015;194(2):575-583.

10. Liu JZ, et al. Zinc sequestration by the neutrophil protein calprotectin enhances Salmonella growth in the inflamed gut. Cell Host Microbe. 2012;11(3):227-239.

11. Loser K, et al. The Toll-like receptor 4 ligands Mrp8 and Mrp14 are crucial in the development of autoreactive CD8+ T cells. Nat Med. 2010;16(6):713-717.

12. van Lent PL, et al. Myeloid-related proteins S100A8/S100A9 regulate joint inflammation and cartilage destruction during antigen-induced arthritis. Ann Rheum Dis. 2008;67(12):1750-1758.

13. Manitz MP, et al. Loss of S100A9 (MRP14) results in reduced interleukin-8-induced CD11b surface expression, a polarized microfilament system, and diminished responsiveness to chemoattractants in vitro. Mol Cell Biol. 2003;23(3):1034-1043.

14. Hobbs JA, et al. Myeloid cell function in MRP-14 (S100A9) null mice. Mol Cell Biol. 2003;23(7):2564-2576.

15. Ehrchen JM, Sunderkötter C, Foell D, Vogl T, Roth J. The endogenous Toll-like receptor 4 agonist S100A8/S100A9 (calprotectin) as innate amplifier of infection, autoimmunity, and cancer.
JLeukoc Biol. 2009;86(3):557-566.

16. Strupat K, Rogniaux H, Van Dorsselaer A, Roth J, Vogl T. Calcium-induced noncovalently linked tetramers of MRP8 and MRP14 are confirmed by electrospray ionization-mass analysis. J Am Soc Mass Spectrom. 2000;11(9):780-788.

17. Leukert N, Vogl T, Strupat K, Reichelt R, Sorg C, Roth J. Calcium-dependent tetramer formation of S100A8 and S100A9 is essential for biological activity. J Mol Biol. 2006;359(4):961-972.

18. Taylor GA, et al. A pathogenetic role for TNF alpha in the syndrome of cachexia, arthritis, and autoimmunity resulting from tristetraprolin (TTP) deficiency. Immunity. 1996;4(5):445-454

19. Pasparakis M, Alexopoulou L, Episkopou V, Kollias G. Immune and inflammatory responses in TNF alpha-deficient mice: a critical requirement for TNF alpha in the formation of primary B cell follicles, follicular dendritic cell networks and germinal centers, and in the maturation of the humoral immune response. J Exp Med. 1996;184(4):1397-1411.

20. Wang $\mathrm{H}$, et al. Activated macrophages are essential in a murine model for $\mathrm{T}$ cell-mediated chronic psoriasiform skin inflammation. J Clin Invest. 2006;116(8):2105-2114.

21. Di Cesare A, Di Meglio P, Nestle FO. The IL-23/ Th17 axis in the immunopathogenesis of psoriasis. J Invest Dermatol. 2009;129(6):1339-1350.

22. Tortola L, et al. Psoriasiform dermatitis is driven by IL-36-mediated DC-keratinocyte crosstalk. J Clin Invest. 2012;122(11):3965-3976.

23. Di Meglio P, Villanova F, Nestle FO. Psoriasis. Cold Spring Harb Perspect Med. 2014;4(8):a015354.

24. Bollmann F, et al. Endothelial dysfunction in tristetraprolin-deficient mice is not caused by enhanced tumor necrosis factor- $\alpha$ expression. J Biol Chem. 2014;289(22):15653-15665.

25. Molle C, et al. Tristetraprolin regulation of interleukin 23 mRNA stability prevents a spontaneous inflammatory disease. J Exp Med. 2013;210(9):1675-1684.

26. Retser E, et al. Doxycycline-induced expression of transgenic human tumor necrosis factor $\alpha$ in adult mice results in psoriasis-like arthritis. Arthritis Rheum. 2013;65(9):2290-2300.

27. Vogl T, et al. Alarmin S100A8/S100A9 as a biomarker for molecular imaging of local inflammatory activity. Nat Commun. 2014;5:4593.

28. Korndörfer IP, Brueckner F, Skerra A. The crystal structure of the human (S100A8/S100A9)2 heterotetramer, calprotectin, illustrates how conformational changes of interacting alpha-helices can determine specific association of two EF- hand proteins. JMol Biol. 2007;370(5):887-898.

29. Itou $\mathrm{H}$, et al. The crystal structure of human MRP14 (S100A9), a Ca(2+)-dependent regulator protein in inflammatory process. J Mol Biol. 2002;316(2):265-276.

30. Frosch M, et al. Myeloid-related proteins 8 and 14 are specifically secreted during interaction of phagocytes and activated endothelium and are useful markers for monitoring disease activity in pauciarticular-onset juvenile rheumatoid arthritis. Arthritis Rheum. 2000;43(3):628-637.

31. Rammes A, Roth J, Goebeler M, Klempt M, Hartmann M, Sorg C. Myeloid-related protein (MRP) 8 and MRP14, calcium-binding proteins of the S100 family, are secreted by activated monocytes via a novel, tubulin-dependent pathway. J Biol Chem. 1997;272(14):9496-9502.

32. Pruenster M, et al. Extracellular MRP8/14 is a regulator of $\beta 2$ integrin-dependent neutrophil slow rolling and adhesion. Nat Commun. 2015;6:6915.

33. Vogl T, Gharibyan AL, Morozova-Roche LA. Pro-inflammatory S100A8 and S100A9 proteins: self-assembly into multifunctional native and amyloid complexes. Int J Mol Sci. 2012;13(3):2893-2917.

34. Pröpper C, Huang X, Roth J, Sorg C, Nacken W. Analysis of the MRP8-MRP14 protein-protein interaction by the two-hybrid system suggests a prominent role of the $\mathrm{C}$-terminal domain of S100 proteins in dimer formation. J Biol Chem 1999;274(1):183-188.

35. Vogl T, Leukert N, Barczyk K, Strupat K, Roth J. Biophysical characterization of S100A8 and S100A9 in the absence and presence of bivalent cations. Biochim Biophys Acta. 2006;1763(11):1298-1306.

36. Ishikawa K, Nakagawa A, Tanaka I, Suzuki M, Nishihira J. The structure of human MRP8, a member of the $\mathrm{S} 100$ calcium-binding protein family, by MAD phasing at 1.9 A resolution. Acta Crystallogr D Biol Crystallogr. 2000;56(Pt 5):559-566.

37. Leukert N, Sorg C, Roth J. Molecular basis of the complex formation between the two calcium-binding proteins S100A8 (MRP8) and S100A9 (MRP14). Biol Chem. 2005;386(5):429-434.

38. Foell D, et al. Methotrexate withdrawal at 6 vs 12 months in juvenile idiopathic arthritis in remission: a randomized clinical trial. JAMA. 2010;303(13):1266-1273.

39. Broome AM, Ryan D, Eckert RL. S100 protein subcellular localization during epidermal differentiation and psoriasis. J Histochem Cytochem. 2003;51(5):675-685. 
40. Schonthaler HB, et al. S100A8-S100A9 protein complex mediates psoriasis by regulating the expression of complement factor C3. Immunity. 2013;39(6):1171-1181.

41. Hiratsuka S, et al. The S100A8-serum amyloid A3-TLR4 paracrine cascade establishes a pre-metastatic phase. Nat Cell Biol. 2008;10(11):1349-1355.

42. Hiratsuka S, et al. Primary tumours modulate innate immune signalling to create pre-metastaic vascular hyperpermeability foci. Nat Commun. 2013;4:1853.

43. Kostakis ID, Cholidou KG, Vaiopoulos AG, Vlachos IS, Perrea D, Vaos G. Fecal calprotectin in pediatric inflammatory bowel disease: a systematic review. Dig Dis Sci. 2013;58(2):309-319.

44. Björk P, et al. Identification of human S100A9 as a novel target for treatment of autoimmune disease via binding to quinoline-3-carboxamides. PLoS Biol. 2009;7(4):e97.

45. Comi G, et al. Effect of laquinimod on MRI- monitored disease activity in patients with relapsingremitting multiple sclerosis: a multicentre, randomised, double-blind, placebo-controlled phase IIb study. Lancet. 2008;371(9630):2085-2092.

46. Qin H, et al. Generation of a new therapeutic peptide that depletes myeloid-derived suppressor cells in tumor-bearing mice. Nat Med. 2014;20(6):676-681.

47. Korb-Pap A, et al. Early structural changes in cartilage and bone are required for the attachment and invasion of inflamed synovial tissue during destructive inflammatory arthritis. Ann Rheum Dis. 2012;71(6):1004-1011.

48. Roth J, Burwinkel F, van den Bos C, Goebeler M, Vollmer E, Sorg C. MRP8 and MRP14, S-100-like proteins associated with myeloid differentiation, are translocated to plasma membrane and intermediate filaments in a calcium-dependent manner. Blood. 1993;82(6):1875-1883.

49. Tscharntke M, et al. Impaired epidermal wound healing in vivo upon inhibition or deletion of
Rac1. JCell Sci. 2007;120(Pt 8):1480-1490.

50. Vogl T, Roth J, Sorg C, Hillenkamp F, Strupat K. Calcium-induced noncovalently linked tetramers of MRP8 and MRP14 detected by ultraviolet matrix-assisted laser desorption/ionization mass spectrometry. JAm Soc Mass Spectrom. 1999;10(11):1124-1130.

51. Ahmad-Nejad P, Häcker H, Rutz M, Bauer S, Vabulas RM, Wagner H. Bacterial CpG-DNA and lipopolysaccharides activate Toll-like receptors at distinct cellular compartments. Eur J Immunol. 2002;32(7):1958-1968.

52. Bosisio D, et al. Stimulation of toll-like receptor 4 expression in human mononuclear phagocytes by interferon-gamma: a molecular basis for priming and synergism with bacterial lipopolysaccharide. Blood. 2002;99(9):3427-3431.

53. Comeau SR, Gatchell DW, Vajda S, Camacho CJ. ClusPro: an automated docking and discrimination method for the prediction of protein complexes. Bioinformatics. 2004;20(1):45-50. 